\title{
Torsion and bending of nucleic acids studied by subnanosecond time-resolved fluorescence depolarization of intercalated dyes
}

\author{
D. P. Millar, ${ }^{\text {a) }}$ R. J. Robbins, and A. H. Zewail')

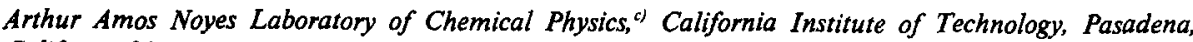 \\ California 91125 \\ (Received 28 August 1981; accepted 15 October 1981)
}

\begin{abstract}
Subnanosecond time-resolved fluorescence depolarization has been used to monitor the reorientation of ethidium bromide intercalated in native DNA, synthetic polynucleotide complexes, and in supercoiled plasmid DNA. The fluorescence polarization anisotropy was successfully analyzed with an elastic model of DNA dynamics, including both torsion and bending, which yielded an accurate value for the torsional rigidity of the different DNA samples. The dependence of the torsional rigidity on the base sequence, helical structure, and tertiary structure was experimentally observed. The magnitude of the polyelectrolyte contribution to the torsional rigidity of DNA was measured over a wide range of ionic strength, and compared with polyelectrolyte theories for the persistence length. We also observed a rapid initial reorientation of the intercalated ethidium which had a much smaller amplitude in RNA than in DNA.
\end{abstract}

\section{INTRODUCTION}

Time-dependent fluorescence depolarization is a powerful technique for the study of molecular reorientation, and has been widely applied on both the nanosecond ${ }^{1,2}$ and picosecond ${ }^{3,4}$ time scales. For rigid molecules, such experiments have provided considerable information about molecular size, conformation, and the strength of solute-solvent interactions. A1though fluorescence depolarization can also be used to study internal motions of flexible molecules, which are relevant, for example, to photoisomerization and the function of many biological macromolecules, few timeresolved experiments have been reported to date. ${ }^{5-8}$ In this paper, we present a full account of earlier communications ${ }^{7,15}$ on DNA and RNA. Specifically, we pursue a systematic study of the rapid internal motions of nucleic acids by means of time-dependent fluorescence depolarization of intercalated dyes.

Studies of the internal motions of nucleic acids are important as a probe of their structure and conformational flexibility. Many of the large-scale structural features of duplex DNA, such as supercoiling and packaging in chromosomes and bacteriophage heads, can be accounted for by elastic models wherein DNA is completely characterized by its bending and torsional rigidities. ${ }^{9-12}$ However, the smooth, continuous deformations of the helix implied by the elastic model are inadequate to explain some observations. For example, the ability of the hydrogen-bonded ring protons of the bases in DNA to exchange at temperatures well below the denaturation point ${ }^{13}$ suggests that the double helix contains a small number of unpaired bases at room temperature. Torsional fluctuations that lead to a transient local unwinding of the helix are also thought to be involved in many important biological events. ${ }^{14}$ In these cases, details of the local helix structure should be important,

a) Fulbright-Hays G raduate Student.

b) Alfred P. Sloan Fellow and Camille and Henry Dreyfus Teacher-Scholar.

${ }^{c)}$ Contribution No. 6503. and a description of DNA solely in terms of its torsional and bending rigidities inadequate. Although the bending rigidity can be deduced from the experimental persistence length, relatively little is known about the torsional rigidity or local torsional fluctuations of the DNA helix. It is these torsional motions that we expect to observe by the fluorescence depolarization technique.

In a pioneering experiment, Wahl et al. ${ }^{5}$ detected a rapid ( $\approx 28 \mathrm{~ns}$ ) decay of the fluorescence polarization anisotropy of ethidium bromide intercalated in DNA, which they ascribed to local oscillatory motions of the bases about the helix axis. Barkely and Zimm ${ }^{11}$ subsequently developed an elastic model of DNA dynamics. They showed ${ }^{11}$ that the decay of the fluorescence polarization anisotropy of an intercalated dye should be complex, containing exponentials in $t^{1 / 2}$ due to torsion and in $t^{1 / 4}$ due to bending, and that the observed depolarization ${ }^{5}$ arose primarily from torsional motions. However, the limited time resolution $(\approx 5 \mathrm{~ns}$ ) of the experimental data then available made a rigorous test of the model impossible, and a precise value for the torsional rigidity could not be obtained.

It has recently been shown ${ }^{7,8}$ that the subnanosecond time-correlated single photon counting technique can provide very accurate fluorescence depolarization data for the ethidium bromide-DNA system with a time resolution of 250 ps. Using this technique, we were able to verify the $\exp \left(-t^{1 / 2}\right)$ torsional relaxation predicted by the BarkleyZimm model, and to obtain an accurate value for the torsional rigidity of our calf thymus DNA sample $C=1.3$ $\pm 0.2 \times 10^{-19}$ erg $\mathrm{cm}$ in $0.15 \mathrm{M} \mathrm{NaCl}$ at $23^{\circ} \mathrm{C}$ ignoring the small bending motions. ${ }^{7}$ In independent studies on viral $\phi 29$ DNA in $0.01 \mathrm{M} \mathrm{NaCl}$, Thomas et al. ${ }^{8}$ found similar results. In this paper, we seek to extend our earlier work on the internal motions of nucleic acids by considering the following, additional points: (1) the effect of small bending motions on the observed fluorescence depolarization; (2) the dependence of torsional rigidity on base sequence and helical structure in a series of model polynucleotides; and (3) the magnitude of the polyelectrolyte contribution to the torsional rigidity of DNA. 


\section{THEORETICAL CONSIDERATIONS}

In fluorescence depolarization experiments, a linearly polarized light pulse preferentially excites those molecules whose transition dipoles are nearly parallel to the electric field of the exciting light. This results in an initial polarization of the fluorescence, which decays in time due to rotational Brownian motion. The depolarization is conveniently represented by the timedependent fluorescence polarization anisotropy $r(t)$, defined by ${ }^{1}$

$$
r(t)=\left[i_{\|}(t)-i_{\perp}(t)\right] /\left[i_{\|}(t)+2 i_{\perp}(t)\right],
$$

where $i_{11}(t)$ and $i_{1}(t)$ are the observed fluorescence intensities polarized parallel and perpendicular, respectively, to the polarization of the exciting light. For an asymmetric rigid molecule, $r(t)$ consists of a sum of five exponentials whose decay times depend solely on the principal diffusion coefficients. ${ }^{1,16}$ However, for a nonrigid molecule, the anisotropy will have additional contributions from internal motions whose decay times depend on the flexibility.

In our experiments, the internal motions of nucleic acids are monitored by the time-dependent fluorescence depolarization of intercalated dyes. The particular dye that we have chosen (ethidium bromide) binds strongly to double-stranded nucleic acids ${ }^{17,18}$ by intercalation between adjacent base pairs such that the plane of the phenanthridinium ring (which contains the transition dipole) makes an angle of about $70^{\circ}$ with the helix axis. ${ }^{19}$ Presumably, the dye is rigidly bound and thus reports local motions of the helix. The double-stranded nucleic acids used in this work are very long $\left(\sim 10^{3}-10^{4}\right.$ base pairs) while the duplex structure confers stiffness on the helix, as measured by its persistence length of about $525 \AA$ [see Sec. IV B]. Rotation of the entire molecule and long-range segmental motions are therefore slow, at least on the order of microseconds, and cannot contribute to the depolarization observed on the nanosecond time scale. What we do expect to see in the fluorescence depolarization experiments are rapid torsional motions of the helix, with a smaller contribution from bending and perhaps a limited wobbling of the dye within its intercalation site.

The connection between the fluorescence depolarization experiments and the torsion and bending dynamics of DNA has been analyzed in detail by Barkley and Zimm. ${ }^{11}$ Here we briefly outline their approach and their main results. According to their model, the DNA molecule is treated as a thin, uniformly flexible rod of length $2 L$ and circular cross section of radius $b$, immersed in a viscous fluid at thermal equilibrium. Dynamical equations for torsion and bending of the rod are generated using results from classical elasticity and hydrodynamic theories. They derive the rotational diffusion equation in normal coordinates and solve it for arbitrary initial amplitudes of the normal modes. This general solution is then adapted to the initial conditions of the fluorescence depolarization experiment, and the resulting time-dependent distribution function for the orientation of the intercalated dye is used to compute the fluorescence polarization anisotropy. Barkley and
Zimm's final expression for the anisotropy can be written in the form ${ }^{11}$

$$
\begin{aligned}
r(t)= & r_{0}\left[A_{4}(\epsilon)+A_{5}(\epsilon) e^{-\Delta}\right]^{-1}\left\{A_{1}(\epsilon) e^{-\Delta}\right. \\
& \left.+A_{2}(\epsilon)\left[5 / 8+3 / 8 e^{-\Delta}\right] e^{-\Sigma}+A_{3}(\epsilon) e^{-\Delta} e^{-5 / 4}\right\},
\end{aligned}
$$

where $r_{0}$ is the limiting anisotropy (which has a theoretical maximum value of 0.4$), \Gamma$ and $\Delta$ are the torsion and bending decay functions, respectively, and the $A_{j}(\epsilon)$ are simple trigonometric functions of the angle $\epsilon$ between the transition dipole of the intercalated dye and the helix axis

$$
\begin{aligned}
& A_{1}(\epsilon)=\frac{1}{4}\left(1-3 \cos ^{2} \epsilon\right)^{2}, \\
& A_{2}(\epsilon)=\frac{3}{4} \sin ^{4} \epsilon, \\
& A_{3}(\epsilon)=3 \sin ^{2} \epsilon \cos ^{2} \epsilon, \\
& A_{4}(\epsilon)=\frac{3}{4}\left(1+\cos ^{2} \epsilon\right), \\
& A_{5}(\epsilon)=\frac{1}{4}\left(1-3 \cos ^{2} \epsilon\right) .
\end{aligned}
$$

The torsion decay function is given by ${ }^{11}$

$$
\Gamma(t)=4 D_{1} t+\left(4 k_{B} T / C L\right) \sum_{k=1}^{\infty}\left[1-\exp \left(-t / \tau_{k}\right)\right] / \lambda_{k}^{2},
$$

which describes a superposition of relaxations due to all torsional normal modes of the rod, with relaxation times $\tau_{k}$ and wave numbers $\lambda_{k}=k \pi / L$, plus a term corresponding to rotation of the entire rod about its long axis, with diffusion coefficient $D_{1} ; C$ is the torsional rigidity of the rod, $k_{B}$ is Boltzmann's constant, and $T$ is the absolute temperature. The torsional relaxation times are

$$
\tau_{k}=\rho / C \lambda_{k}^{2},
$$

where $\rho$ is the rotational friction coefficient per unit length

$$
\rho=4 \pi \eta b^{2},
$$

and $\eta$ is the solvent viscosity. A similar analysis for bending gives ${ }^{11}$

$$
\Delta(t)=4 D_{2} t+\left(4 k_{B} T / E I L\right) \sum_{k \text { oren }}^{\infty}\left[1-\exp \left(-t / \tau_{k}^{\prime}\right)\right] / \kappa_{k}^{2},
$$

which describes a superposition of relaxations due to bending normal modes of the rod, with relaxation times $\tau_{k}^{\prime}$ and wave numbers $\kappa_{k} \simeq(2 k+1) \pi / 4 L$, plus a term cor responding to rotation of the entire rod about a transverse axis, with diffusion coefficient $D_{2} ; E I$ is the bending rigidity of the rod. The bending relaxation times are

$$
\tau_{k}^{\prime}=\rho_{k} / E I \kappa_{k}^{2},
$$

where the friction coefficients for bending $\rho_{k}$ depend on $k$ as

$$
\rho_{k}=4 \pi \eta / \kappa_{k}^{2} f\left(\kappa_{k} b\right),
$$

with $f(z)$ a sum of modified Bessel functions

$$
f(z)=K_{0}(z)+(z / 2) K_{1}(z) \text {. }
$$

For certain times in the decay process, the cumbersome expressions for $\Gamma$ and $\Delta[$ Eqs. (4) and (7), respectively] can be approximated by simple expressions. At sufficiently short times, the discrete sums can be replaced by integrals via the Euler-Marclaurin summa- 
TABLE I. Fluorescence lifetimes of ethidium bromide.

\begin{tabular}{llll}
\hline Sample & $\tau_{f}(\mathrm{~ns})$ & $\tau_{b}(\mathrm{~ns})$ & $f$ \\
\hline Free & $1.8(0.1)^{\mathrm{b}}$ & & \\
$\mathrm{CT}$ & $2.0(1.2)$ & $23.0(0.5)$ & $0.05(0.02)$ \\
$d(\mathrm{G}-\mathrm{C}) \cdot d(\mathrm{G}-\mathrm{C})$ & $2.1(0.2)$ & $23.5(0.2)$ & $0.18(0.04)$ \\
$d(\mathrm{~A}-\mathrm{T}) \cdot d(\mathrm{~A}-\mathrm{T})$ & $2.5(0.8)$ & $25.4(0.4)$ & $0.07(0.02)$ \\
$d \mathrm{G} \cdot d \mathrm{C}$ & $2.4(0.2)$ & $22.2(0.4)$ & $0.18(0.04)$ \\
$d \mathrm{~A} \cdot d \mathrm{~T}$ & $2.3(0.2)$ & $19.9(0.2)$ & $0.31(0.02)$ \\
$d \mathrm{~A} \cdot d \mathrm{U}$ & $2.6(0.4)$ & $22.4(0.9)$ & $0.31(0.06)$ \\
$r \mathrm{~A} \cdot r \mathrm{U}$ & $3.0(0.4)$ & $27.6(0.7)$ & $0.22(0.02)$ \\
$d \mathrm{~A} \cdot r \mathrm{U} \cdot r \mathrm{U}$ & $2.7(0.3)$ & $23.9(0.5)$ & $0.14(0.06)$ \\
$\mathrm{pBR} \mathrm{322}$ & $2.8(1.1)$ & $23.2(0.2)$ & $0.06(0.02)$ \\
\hline
\end{tabular}

aAbbreviations: $C T$ is calf thymus DNA; pBR 322 is supercoiled plasmid DNA; $d(\mathrm{G}-\mathrm{C}) \cdot d(\mathrm{G}-\mathrm{C})$ is poly $d(\mathrm{G}-\mathrm{C})$ - poly $d(\mathrm{G}-\mathrm{C})$; and similarly for the remainder.

Values in parentheses are $95 \%$ confidence intervals.

The quantities $\tau_{f}, \tau_{b}$, and $f$ are defined in Eq。 (16).

tion formula plus its leading correction term and integrated by parts; at very long times, the exponentials vanish and the infinite series can be summed directly. The resulting approximate expressions for $\Gamma$ are

$$
\Gamma(t) \simeq \begin{cases}A t^{1 / 2}, & t \leq \tau_{1}, \\ 4 D_{1} t+2 k_{B} T L / 3 C, & t \gg \tau_{1},\end{cases}
$$

with

$$
A=2 k_{B} T / \pi b \eta^{1 / 2} C^{1 / 2} .
$$

In Eq. (7) for the bending decay function, the Bessel function term appearing in the exponent is treated as a constant for the purposes of integration, and the value of the argument that maximizes the integrand $z_{\max }$ is used to compute this constant. The resulting expressions for $\Delta$ are

$$
\Delta(t) \approx \begin{cases}B(t) t^{1 / 4}, & t \lesssim \tau_{1}^{\prime}, \\ 4 D_{2} t+0.4853 k_{B} T L / E I, & t \gg \tau_{1}^{\prime},\end{cases}
$$

where $B(t)$ is a slowly varying function of time

$$
B(t)=\left(3.466 k_{B} T / \pi^{5 / 4} \eta^{1 / 4} E I^{3 / 4}\right) f\left(z_{\max }\right)^{1 / 4},
$$

and $z_{\max }$ is determined by iteration from the equation

$$
z_{\max }(t) \simeq b\left[5.024 \pi \eta / \text { EIf } f\left(z_{\max }\right)\right]^{1 / 4} .
$$

Barkley and Zimm ${ }^{11}$ have also considered the problem of an initial, very rapid, but limited, reorientation of the transition dipole, caused, for example, by wobbling of the dye within its intercalation site. They show that this initial reorientation can be accounted for by replacing $\Gamma(t)$ in the expression for the anisotropy [Eq. (2)] by $\Gamma(t)+\Xi$, where $\Xi$ is the wobble parameter. The square root of $\Xi$ measures the width of the (Gaussian) distribution of dye orientations after wobbling.

Equation (2) for the fluorescence polarization anisotropy and the short-time approximations for $\Gamma$ and $\Delta$ [Eqs. (11a) and (13a), respectively] constitute the most useful results of this section. They will be applied to the analysis of our experimental data in Sec. IV.

\section{EXPERIMENTAL TECHNIQUES}

\section{A. Samples}

Ethidium bromide and calf thymus DNA were obtained from Sigma and used without further purification. The synthetic polynucleotides (Table I) were obtained from PL Biochemicals and dialyzed against appropriate buffers for $24 \mathrm{~h}$ before use. Supercoiled pBR 322 plasmid DNA was prepared according to standard procedures ${ }^{30}$ and was purified by an additional cycle of density gradientultracentrifuguation. Samples were prepared using triply distilled water (adjusted to $p \mathrm{H} 7.7$ with dilute $\mathrm{NaOH}$ ) or Tris $\cdot \mathrm{HCl} p \mathrm{H} 7.7$ buffer, and the desired ionic strength obtained by adding $\mathrm{NaCl}$. Nucleotide to dye ratios $(P / D)$ were in the range $90: 1$ to $900: 1$. All fluorescence measurements were performed at room temperature $\left(23^{\circ} \mathrm{C}\right)$.

Sedimentation coefficients of the synthetic polynucleotide samples were between 7 and 10 Svedberg, corresponding to molecular weights between $3 \times 10^{5}$ and $1 \times 10^{6}$ dalton. ${ }^{71}$ The molecular weight distributions of the polynucleotides, determined by Sepharose $4 \mathrm{~B}$ column chromatography, indicate the presence of aggregates and hairpins (alternating sequences only) in addition to individual DNA rods. ${ }^{72}$ The double-strand length in the rods is between 500 and 1000 base pairs, in the hairpins is about 500 base pairs, and in the aggregates is several thousand base pairs. ${ }^{72}$ Thus, the minimum double-strand length in any of the synthetic polynucleotides is about 500 base pairs, while the average length is considerably more. Calf thymus DNA is about 10000 base pairs long while pBR 322 DNA is about 4000 base pairs long. All of the nucleic acid samples were sufficiently long that the observed relaxation behavior was not affected by reorientation of the entire DNA chain (these motions for a 500 base pair rod have relaxation times longer than $200 \mathrm{~ns})$. All of the samples are expected to exhibit length-independent relaxation dynamics described by Egs. (11a) and (13a), since these equations are valid over the entire fluorescence decay for DNA chains longer than about 200 base pairs.

\section{B. Time-resolved fluorescence measurements}

Time-resolved fluorescence measurements were performed using the single photon counting apparatus shown in Fig. 1. The actively mode-locked argon ion laser (Spectra Physics model 171/342) produced a train of $100 \mathrm{ps}$ pulses at a repetition frequency of $82.190 \mathrm{MHz}$, with an average power of $900 \mathrm{~mW}$ at $5145 \AA$. Excitation pulses were sampled at a frequency of $250 \mathrm{kHz}$ by an acousto-optic modulator (Spectra Physics model 365 with model $465 \mathrm{rf}$ driver) synchronized to the laser output. Synchronization was achieved by focusing a small portion of the laser output onto a fast photodiode (Hew lett-Packard 5082-4203), gating the resulting electrical pulses at the desired frequency with a fast linear gate and discriminator combination (ORTEC models LG 101 and 436), and then using the gated pulses to trigger the rf driver via a variable delay (Fig. 1). After careful optical alignment and electrical synchronization, a contrast ratio between the main pulse and subsidiary pulses of better than $300: 1$ was routinely obtained. The 


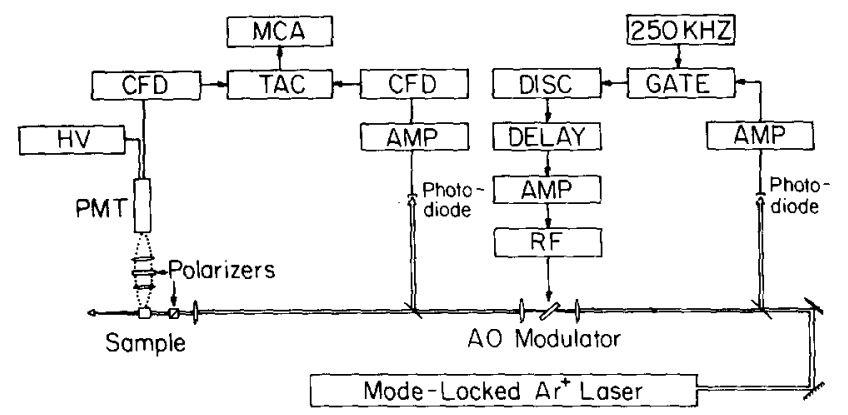

FIG. 1. Experimental setup for time-correlated single photon counting. PMT, Amperex XP2020Q photomultiplier; HV, high voltage power supply; CFD, constant fraction discriminator; DISC, discriminator; TAC, time to amplitude converter; MCA, multichannel analyzer; GATE, fast linear gate; $250 \mathrm{kHz}$, pulse generator; RF, driver for acousto-optic modulator.

excitation pulses were attenuated by a factor of about 1000 and passed through a vertical polarizer before being focused into the sample.

The "start" signal for the time-to-amplitude converter (TAC, ORTEC model 457) was obtained from a portion of the laser output via a second fast photodiode and a constant fraction discriminator (ORTEC model 473 A). Fluorescence emitted at right angles to excitation was detected by an Amperex XP2020Q photomultiplier; its output was fed into a constant fraction differential discriminator (ORTEC model 583), which provided the "stop" signal for the TAC. The excitation beam was attenuated so that the ratio of stop to start pulses was always less than $1 \%$. The TAC outputs were processed and stored in a Tracor-Northern TN-1706 multichannel pulse-height analyzer, which was interfaced to a PDP $11 / 23$ computer.

The fluorescence components polarized parallel $i_{\| \prime}(t)$ and perpendicular $i_{1}(t)$ to the direction of polarization of the exciting light were selected by a rotatable polarizer and acquired alternately in separate halves of the memory of the multichannel analyzer for equal total analyzer lifetimes (4-10000 s each). Cutoff filters (Schott OG570 and OG590) were placed in front of the photomultiplier to isolate emission at wavelengths longer than $590 \mathrm{~nm}$ and remove scattered laser light. A calcite depolarizer was also placed in front of the photomultiplier to avoid any polarization bias in its response. The fluorescence decay law $s(t)$ could be obtained, independent of molecular reorientation, by setting the emission polarizer at $54.7^{\circ}$ to the direction of parallel polarization. ${ }^{3}$ The instrument response function obtained by scattering the laser pulses from a dilute suspension of coffee creamer had a width of $250 \mathrm{ps}$.

\section{Data analysis}

In the experiments reported here, the instrument response function was sufficiently narrow $(250 \mathrm{ps})$ that the observed fluorescence decays were not distorted by convolution. The fluorescence decay law $s(t)$, obtained by setting the emission polarizer at $54.7^{\circ}$ to the direction of parallel polarization, was fit to a sum of exponentials

$$
s(t)=A\left[f \exp \left(-t / \tau_{f}\right)+(1-f) \exp \left(-t / \tau_{b}\right)\right],
$$

where $A$ was the amplitude, $\tau_{f}$ and $\tau_{b}$ were the lifetimes of the nonintercalated ("free") and intercalated ("bound") dye, respectively, and $f$ was the fraction of nonintercalated dye. For the analysis of the fluorescence depolarization data, we first formed the experimental sum and difference curves

$$
\begin{aligned}
& s(t)=i_{11}(t)+2 i_{\perp}(t), \\
& d(t)=i_{11}(t)-i_{\perp}(t),
\end{aligned}
$$

respectively. These functions were taken to be of the form

$$
\begin{aligned}
& s(t)=A \exp \left(-t / \tau_{b}\right), \\
& d(t)=r(t) \cdot s(t),
\end{aligned}
$$

with the fluorescence polarization anisotropy $r(t)$ given by Eqs. (2), (11a), and (13a). Of the quantities appearing in the expression for the anisotropy, the helix radius $b$, the flexural rigidity $E I$, the dye tilt $\epsilon$, and the limiting anisotropy $r_{0}$ were estimated from available data (Sec. IV), while the torsional rigidity $C$ and the wobble parameter $\Xi$ were treated as adjustable parameters. The values of the four parameters $A, \tau_{b}, C$, and $\Xi$ were then adjusted so as to simultaneously obtain the best fit of the experimental $s(t)$ and $d(t)$ curves to the model defined by Eq. (18). In samples where there was a significant fraction of free ethidium $\left(\tau_{f} \approx 1.8 \mathrm{~ns}\right)$ it was necessary to ignore the first $5 \mathrm{~ns}$ of the decays in the fitting procedure. The curve fitting was performed on a PDP 11/23 computer using a nonlinear least-squares routine based on Marquardt's algorithm. ${ }^{20,21}$ The quality of the fits was judged by the reduced chi-squared $\left(\chi_{r}^{2}\right)$ criterion ${ }^{21}$ and by inspection of the weighted residuals and the residual autocorrelation function. ${ }^{22}$

\section{RESULTS}

\section{A. Fluorescence lifetimes}

The fluorescence decays of ethidium bromide in aqueous solution $(1 \mu \mathrm{g} / \mathrm{ml}$ in $0.1 \mathrm{M}$ Tris $\cdot \mathrm{HCl}, p \mathrm{H} 7.7 / 0.15$ $\mathrm{M} \mathrm{NaCl})$ and bound to the nucleic acids $(100 \mu \mathrm{g} / \mathrm{ml}$ nucleic acid, $P / D \simeq 120$ ) were measured by setting the emission polarizer at $54.7^{\circ}$ to the direction of polarization of the exciting light (Table I). A typical fluorescence decay curve, for the ethidium-calf thymus DNA system, is shown in Fig. 2.

For free ethidium bromide, the fluorescence decay was exponential with a lifetime of $1.8 \mathrm{~ns}$. Upon binding to the nucleic acids there was a striking increase in the fluorescence lifetime of ethidium bromide (Table I), which provided a sensitive test for intercalation. However, for none of our samples was a purely single exponential decay observed: in each case the data could be fit to a sum of two exponentials [Eq. (16)] with lifetimes of approximately $2-3$ and $20-27 \mathrm{~ns}$. The lifetime of the shorter component $(\sim 2-3 \mathrm{~ns})$ was similar to that measured for free ethidium bromide $(1.8 \mathrm{~ns})$. However, due to the small fraction of this component and the resulting uncertainty in its lifetime, we could not exclude the possibility of a contribution from an electrostatically bound complex ${ }^{18}$ with a lifetime intermediate between those of the free and intercalated dye. The two components were 


$$
\begin{array}{r}
-0 \\
3 \\
0 \\
0 \\
0 \\
0
\end{array}
$$$$
\begin{gathered}
m \\
1 \\
0 \\
x \\
> \\
+ \\
- \\
0 \\
5 \\
0 \\
+1 \\
5 \\
1-1
\end{gathered}
$$

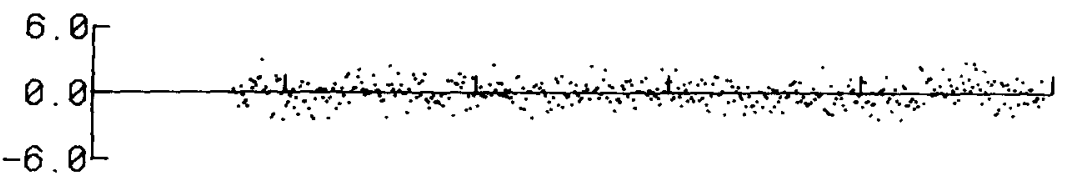

$m$
1
0
$x$
$>$
+
0
0
0
0
+5
1
+-1

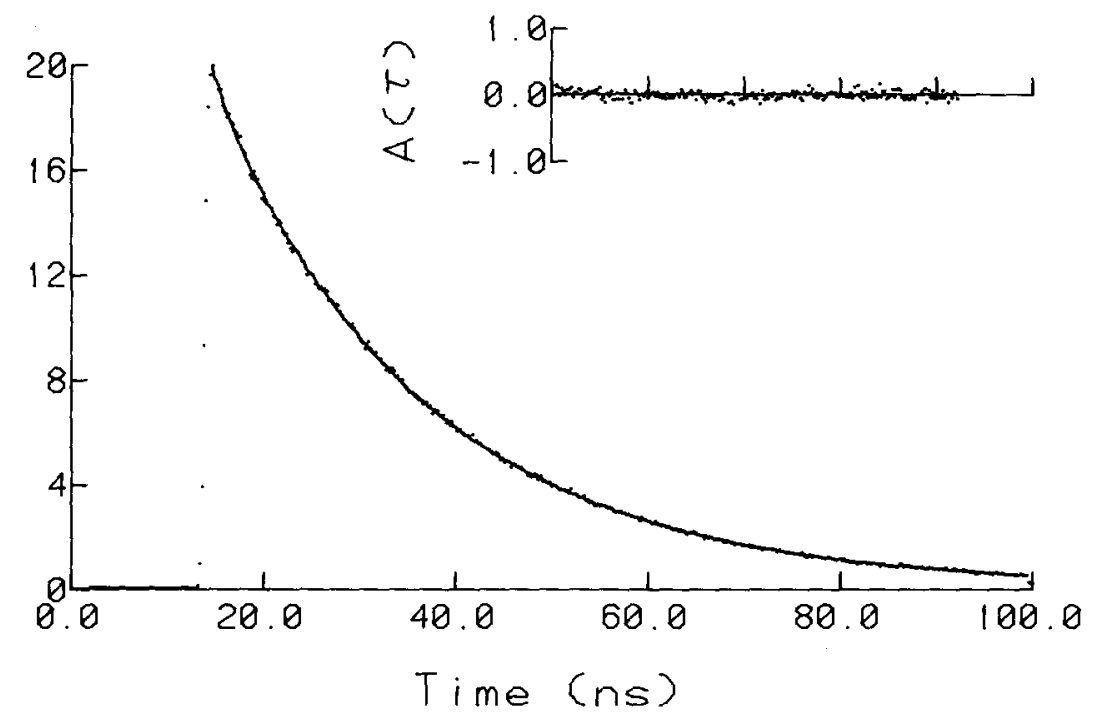

FIG. 2. Isotropic fluorescence decay $(54.7)$ of the ethidium: calf thymus DNA complex in aqueous Tris

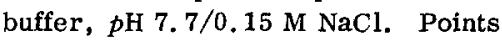
are experimental data. The smooth line is the best fit to Eq. (16) with $\tau_{f}=2.00 \pm 1.2 \mathrm{~ns}, \tau_{b}=22.38 \pm 0.20 \mathrm{~ns}$, $f=0.062 \pm 0.027$, and $\chi_{r}^{2}=1.078$. The weighted residuals and their autocorrelation function $A(\tau)$ are also shown (the time scale along the $\tau$ axis is 0 to $50 \mathrm{~ns}$ ). therefore assigned to nonintercalated and intercalated dye species, respectively. The fraction $f$ of the shorter component thus provided a measure of the extent of ethidium intercalcation, which was important for the analysis of the fluorescence depolarization data since the model for $r(t)$ [Eq. (2)] applies only to the intercalated dye.

For the ethidium-calf thymus DNA sample, the lifetime of the intercalated dye was $23.0 \mathrm{~ns}$, with $5 \%$ of nonintercalated dye. Similar results were obtained for ethidium bromide bound to the supercoiled pBR 322 plasmid DNA, whereas there was consideralbe variation among the synthetic polynucleotides (Table I). The lifetime of the intercalated ethidium ranged from 19.9 $\mathrm{ns}$, when bound to poly $(d \mathrm{~A}) \cdot \operatorname{poly}(d \mathrm{~T})$, to $27.6 \mathrm{~ns}$, when bound to poly $(r \mathrm{~A}) \cdot \operatorname{poly}(r \mathrm{U})$, while the fraction of nonintercalated dye ranged from $7 \%$ for poly $d(\mathrm{~A}-\mathrm{T}) \cdot$ poly $d(\mathrm{~A}-\mathrm{T})$ to $31 \%$ for poly $(d \mathrm{~A}) \cdot \operatorname{poly}(d \mathrm{U})$. Despite these variations, several trends emerged: (1) Of the polydeoxyribonucleotides, the homopolymers poly $(d A)$ - poly $(d T)$ and poly $(d G) \cdot \operatorname{poly}(d C)$ had shorter lifetimes and more nonintercalated dye than their alternating copolymer counterparts poly $d(\mathrm{~A}-\mathrm{T}) \cdot$ poly $d(\mathrm{~A}-\mathrm{T})$ and poly $d(\mathrm{G}-\mathrm{C}) \cdot$ poly $d(\mathrm{G}-\mathrm{C})$; this effect was much larger for $A-T$ than for $G-C$ containing polynucleotides. (2) Ethidium bromide had a significantly longer lifetime, and there was less nonintercalated dye, when bound to the polyribonucleotide poly $(r A) \cdot \operatorname{poly}(r \mathrm{U})\left(\tau_{b}=27.6\right.$ ns, $f=0.22)$ than when bound to its deoxy counterpart poly $(d \mathrm{~A}) \cdot \operatorname{poly}(d \mathrm{U})\left(\tau_{b}=22.4 \mathrm{~ns}, f=0.31\right)$. (3) In series of related polynucleotides, there was a strong correlation between the fluorescence lifetime of the intercalated dye and the extent of binding, long lifetimes being associated with small fractions of nonintercalated dye.
Changes in the nucleotide to dye ratio within the range $P / D \simeq 90-900$, at constant nucleic acid concentration, had no measurable effect on the fluorescence decays. However, the fraction of nonintercalated dye was found to depend on the nucleic acid concentration and ionic strength in a fairly simple manner. In a series of experiments on the ethidium-calf thymus DNA system (0.05 M Tris $\cdot \mathrm{HCl}, p \mathrm{H} 7.7 / 0.15 \mathrm{M} \mathrm{NaCl} ; P / D \simeq 300)$, the fraction of nonintercalated dye increased from $4 \%$ at a DNA concentration of $250 \mu \mathrm{g} / \mathrm{ml}$ to over $35 \%$ at $8 \mu \mathrm{g} / \mathrm{ml}$. The observed concentration dependence was in accord with the neighbor exclusion model of ethidium binding ${ }^{23}$ with an intrinsic binding constant of about 6 $\times 10^{4} \mathrm{M}^{-1}$. In another series of experiments on the ethidium-calf thymus DNA system $(250 \mu \mathrm{g} / \mathrm{ml}$ in water, $p \mathrm{H} 7.7 ; P / D \simeq 300)$, the fraction of nonintercalated dye decreased from $12 \%$ at $1 \mathrm{M} \mathrm{NaCl}$ to $3 \%$ at a salt concentration of $10^{-3} \mathrm{M}$. The observation of a decrease in binding at higher salt concentrations was consistent with both competitive binding of ethidium and sodium cations to $\mathrm{DNA}^{18}$ and the counterion condensation theory, ${ }^{24,25}$ wherein binding of an ethidium displaces a certain number of $\mathrm{Na}^{+}$counterions.

\section{B. Analysis of fluorescence depolarization data}

Time-resolved fluorescence depolarization studies of ethidium bromide intercalated in the nucleic acids were performed by measuring the polarized emission components $i_{11}(t)$ and $i_{1}(t)$ separately for each sample, and then combining them in the computer. Figure 3 shows a typical set of experimental data, for the ethidiumcalf thymus DNA system $(250 \mu \mathrm{g} / \mathrm{ml}$ DNA in $0.01 \mathrm{M}$ $\mathrm{NaCl}, p \mathrm{H} 7.7 ; P / D \simeq 300$ ), and the fluorescence polarization anisotropy $r(t)$ constructed from these data according to Eq. (1). Experiments were performed on a range 

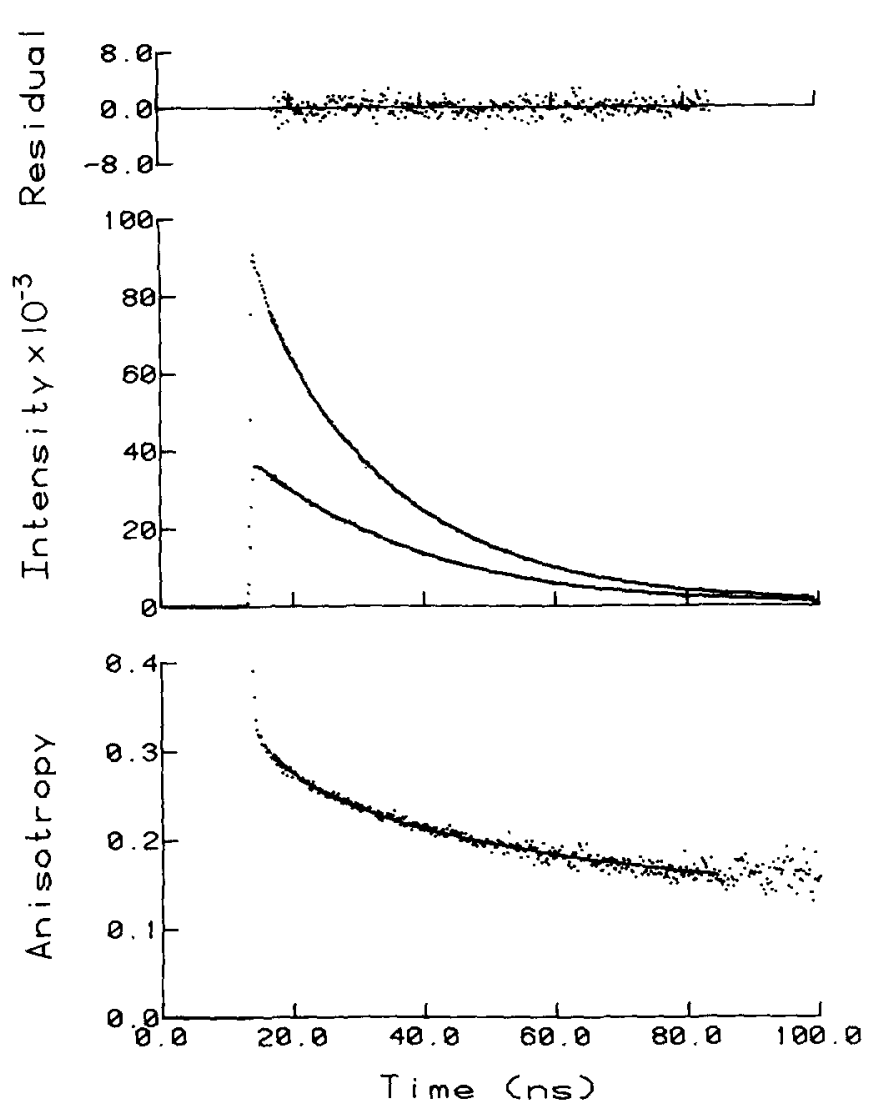

FIG. 3. Experimental $i_{\|}(t)$ and $i_{1}(t)$ curves (center graph) and anisotropy $r(t)$ (lower graph) for the ethidium: calf thymus

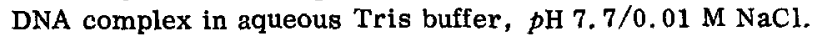
The points are experimental data and the smooth lines are the best fit of Eq. (18) with $\tau_{b}=23.3 \pm 0.05 \mathrm{~ns}, C=1.59 \pm 0.11$ $\times 10^{-19} \mathrm{erg} \mathrm{cm}, \Xi^{1 / 2}=16.3^{\circ} \pm 1.2^{\circ}$, and $\chi_{r}^{2}=1,65$. Also shown (upper graph) are the weighted residuals for the difference curve $i_{11}(t)-i_{1}(t)$.

of nucleic acids to illustrate the effects of helical structure, and on calf thymus DNA as a function of ionic strength to illustrate the polyelectrolyte contribution to the torsional rigidity of DNA. However, before presenting these data, we will explain in some detail how we analyzed the experimental data.

In our earlier work ${ }^{7,15}$ we analyzed our data according to a simplified model, wherein it was assumed that the ethidium transition dipole was perpendicular to the helix axis $\left(\epsilon=90^{\circ}\right)$, that bending motions did not contribute to the observed depolarization $(E I \rightarrow \infty)$, and that the effects of dye wobble could be absorbed into the limiting anisotropy $r_{0}(\Xi=0)$. With these assumptions, the expression for the fluorescence polarization anisotropy reduced to

$$
r(t)=r_{0}\left\{\frac{1}{4}+\frac{3}{4} \exp \left[-\left(2 k_{B} T / \pi\right)\left(t / b^{2} \eta C\right)^{1 / 2}\right]\right\} .
$$

The parameters $r_{0}$ and $b^{2} \eta C$ were then extracted from the data along with the amplitude $A$ and fluorescence lifetime $\tau_{b}$ by the nonlinear least-squares curve-fitting procedure. A similar simplified model was employed by Thomas et al. ${ }^{8}$ except that they did not invoke the restriction $\epsilon=90^{\circ}$. However, many more parameters appear in the general expression for the anisotropy [Eqs. (2), (11a), and (13a)]. Some additional informa- tion must therefore be introduced to reduce the number of adjustable parameters. The procedure we adopted was to estimate the helix radius $b$, the torsional rigidity $E I$, the dye tilt $\epsilon$, and the limiting anisotropy $r_{0}$ from available data, and then to treat the torsional rigidity $C$ and the dye wobble $\Xi$ as adjustable parameters.

The hydrodynamic radius of the native, $B$ form of DNA was estimated by fitting the sedimentation velocities of low molecular weight, homogeneous DNA fragments as a function of length to the theory of Yamakawa and Fujii. ${ }^{26}$ Kovacic and van Holde ${ }^{27}$ have made very accurate measurements of the sedimentation velocities of 16 double-stranded PM2 DNA restriction fragments with sizes covering the range from 50 to 1735 base pairs. From a nonlinear least-squares analysis of their data, we obtained a hydrodynamic radius of $13.4 \pm 0.3$ $\AA$ and a persistence length of $522 \pm 16 \AA$; the fit was excellent $\left(\chi_{r}^{2}=0.96\right)$. The value of $13.4 \AA$ seemed reasonable for the radius of the hydrated double helix when compared with the bare radius of about $11 \AA$ determined from $x$-ray diffraction studies of oriented fibers of both B-DNA and A-RNA. ${ }^{28}$ The hydrodynamic radius of A-RNA was assumed to be the same as that of B-DNA. All of the nucleic acids investigated were double helical, except for $\operatorname{poly}(d \mathrm{~A}) \cdot \operatorname{poly}(r \mathrm{U}) \cdot \operatorname{poly}(r \mathrm{U})$, which had an unusual triple helical structure. ${ }^{29}$ The hydrodynamic radius of the triple helix was estimated to be $14.8 \AA$ by taking the bare radius from the $x$-ray diffraction studies ${ }^{29}(12.4 \AA)$ and adding on the thickness of the hydration layer (2.4 $\AA$ ) inferred from the data for B-DNA.

The bending rigidity $E I$ is directly related to the persistence length $P$ by the formula ${ }^{11}$

$$
E I=k_{B} T P \text {. }
$$

We estimated a persistence length of $522 \pm 16 \AA$ for (native) DNA under standard conditions (neutral aqueous solution, $0.2 \mathrm{M} \mathrm{NaCl}, 20^{\circ} \mathrm{C}$ ) from a nonlinear leastsquares analysis of the sedimentation data of Kovacic and van Holde (see above). Inclusion of the data of Reinert et al. , ${ }^{31}$ Prunell and Bernardi, ${ }^{32}$ Record et al. , ${ }^{33}$ and Godfrey, ${ }^{34}$ which together cover the size range $50-$ 40000 base pairs $\left[\left(3 \times 10^{4}\right)-\left(3 \times 10^{7}\right)\right.$ daltons $]$, in our analysis gave essentially identical results $b=13.3 \pm 0.3 \AA$ and $P=532 \pm 16 \AA$. This corresponds to a bending rigidity of $(2.15 \pm 0.06) \times 10^{-19} \mathrm{erg} \mathrm{cm}$. Our estimate of the persistence length agreed with those determined by light scattering ${ }^{35-38}(490-660 \AA)$, intrinsic viscosity $^{37,39}(500-550 \AA$, with $b \simeq 12 \AA)$, and electron microscopy $^{40}(530 \pm 50 \AA)$. While native DNA's have been well characterized under standard conditions, very little is known about the persistence lengths of the synthetic polynucleotides. There has also been some controversy over the magnitude of the change in the persistence length of DNA with ionic strength. We circumvented these difficulties in the following way: Firstly, we analyzed the fluorescence depolarization data for calf thymus DNA under standard conditions, holding the bending rigidity fixed at $2.15 \times 10^{-19} \mathrm{erg} \mathrm{cm}$. This gave a torsional rigidity $C$ of $1.43 \times 10^{-19} \mathrm{erg} \mathrm{cm}$. According to classical elasticity theory, the torsional and bending rigidities are related through the formula ${ }^{11}$ 


$$
C=E I(1+\sigma),
$$

where $\sigma$ is Poisson's ratio. From the rigidities of calf thymus DNA under standard conditions we deduced $\sigma$ $=0.50$. It was then assumed that this same Poisson ratio applied to all of the nucleic acids, under all conditions, and the bending rigidity of each sample was calculated from Eq. (21) at each iteration of the curvefitting procedure.

The dye-tilt angle $\epsilon$ for the ethidium-calf thymus DNA system was taken directly from the transient electric dichroism studies of Hogan et al. ${ }^{19}$ From the limiting dichroism at high fields, they calculated that the intercalated dye was not perpendicular to the helix axis, but was tilted by about $20^{\circ}$ along its long axis $\left(\epsilon=70^{\circ}\right.$ $\pm 2^{\circ}$ ). From similar dichroism experiments on the DNA bases, ${ }^{42}$ they also estimated that the bases themselves were inclined at an angle of $73^{\circ} \pm 3^{\circ}$ to the helix axis, in agreement with the revised model for B-DNA proposed by Levitt ${ }^{43}$ on the basis of energy-minimization calculations. The polynucleotides poly $(r \mathrm{~A}) \cdot \operatorname{poly}(r \mathrm{U})$ and $\operatorname{poly}(d \mathrm{~A}) \cdot \operatorname{poly}(r \mathrm{U}) \cdot \operatorname{poly}(r \mathrm{U})$ do not adopt the native B-DNA structure. However, we could not find electric dichroism data for either native or synthetic RNA's in the literature, and therefore we used the value $\epsilon=70^{\circ}$, which was the base tilt obtained from $x$-ray fiber diffraction studies of RNA. ${ }^{28}$

In a rigid medium, where Brownian motion is suppressed, the fluorescence polarization anisotropy should approach its theoretical maximal value of 0.4 . The observation of a limiting anisotropy lower than 0.4 can be due to electronic effects within the molecule that lead to an angle between the absorption and emission dipoles, or to some experimental defect, such as imperfect polarization of the exciting light. For the ethidiumcalf thymus DNA complex in saturated sucrose $(\eta \simeq 480$ cP) we did, in fact, obtain $r_{0}=0.39 \pm 0.01$, but the anisotropy decayed within a few nanoseconds to a constant (plateau) value of $0.36 .{ }^{7}$ A limiting anisotropy $r_{0}=0.39$ \pm 0.01 was also obtained for free ethidium bromide in saturated sucrose and in glycerol $(\eta \simeq 1000 \mathrm{cP}){ }^{7}$ This value was used throughout our analysis.

In this section, we have explained how we estimated the hydrodynamic radius $b$, the Poisson ratio $\sigma$, the dye-tilt angle $\epsilon$, and the limiting anisotropy $r_{0}$ for each sample. We are now in a position to analyze the fluorescence depolarization data and thereby extract the torsional rigidities $C$ and dye-wobble angles $\Xi^{1 / 2}$. In

TABLE II. Torsional rigidity of DNA under standard conditions.

\begin{tabular}{llll}
\hline$[\mathrm{DNA}](\mu \mathrm{g} / \mathrm{ml})$ & $P / D$ & $C\left(10^{-19} \mathrm{erg} \mathrm{cm}\right)$ & $\Xi^{1 / 2}(\mathrm{deg})$ \\
\hline 250 & 300 & $1.48(0.14)^{2}$ & $\cdots$ \\
125 & 300 & $1.38(0.25)$ & $\cdots$ \\
64 & 300 & $1.46(0.17)$ & $\cdots$ \\
32 & 300 & $1.56(0.29)$ & $\cdots$ \\
250 & 90 & $1.37(0.14)$ & $17(3)$ \\
250 & 300 & $1.48(0.14)$ & $17(2)$ \\
250 & 900 & $1.51(0.15)$ & $13(3)$ \\
\hline
\end{tabular}

${ }^{\mathrm{a}} \mathrm{V}$ alues in parentheses are $95 \%$ confidence intervals.
TABLE III. Apparent torsional rigidity of DNA as a function of the dye-tilt angle and Poisson ratio.

\begin{tabular}{llll}
\hline$\sigma:$ & $\infty$ & 0.5 & 0 \\
$\epsilon$ & & & \\
\hline 70 & $0.93(0.06)^{\mathrm{a}, \mathrm{b}}$ & $1.43(0.09)$ & $1.59(0.10)$ \\
90 & $1.17(0.07)$ & $1.60(0.10)$ & $1.73(0.11)$
\end{tabular}

${ }^{a}$ Values in parentheses are $95 \%$ confidence intervals.

${ }^{b}$ Torsional rigidities are expressed in units of $10^{-19} \mathrm{erg} \mathrm{cm}$.

the following sections we present the results of these analyses.

\section{Calf thymus DNA under standard conditions}

Table II summarizes the results of some control experiments on the ethidium-calf thymus DNA system under standard conditions (neutral aqueous solution, $\sim 0.2 \mathrm{M} \mathrm{NaCl}, 20^{\circ} \mathrm{C}$ ). Several points were noted from these results: (1) The torsional rigidity was independent of DNA concentration over the range $32-250 \mu \mathrm{g} / \mathrm{ml}$, and therefore our experiments were monitoring a purely intramolecular property of the double helix. At DNA concentrations of less than $32 \mu \mathrm{g} / \mathrm{ml}$, the fraction of nonintercalated dye was too high for reliable analysis of the data. (2) The torsional rigidity was also independent of the nucleotide to dye ratio over the range 90-900; from this observation we inferred that electronic energy transfer between intercalated dyes was not contributing to the depolarization. (3) There appeared to be some slight dependence of the dye-wobble angle of the nucleotide to dye ratio, ranging from about $17^{\circ}$ at $P / D=90$ to about $13^{\circ}$ at $P / D=900$, but this difference may not be significant. (4) There was no dependence of the wobble angle on the fraction of nonintercalated dye; this fraction was varied from $2 \%$ to $35 \%$ by changing the concentrations of salt and DNA, and by binding to different nucleic acids. From the results in Table II, we calculated an average torsional rigidity of $\left(1.43_{ \pm} 0.09\right) \times 10^{-19} \mathrm{erg} \mathrm{cm}$ for calf thymus DNA under standard conditions.

We also analyzed these data using different values for the dye-tilt angle $\epsilon$ and the Poisson ratio $\sigma$ to enable a comparison with our previous work ${ }^{7,15}$ and that of Thomas et al. ${ }^{8}$ The apparent torsional rigidities are given in Table III. Two dye-tilt angles were tried in these calculations, i.e., $\epsilon=70^{\circ}$ and $84^{\circ}-90^{\circ}$, which were the values deduced from transient electric dichroism ${ }^{19}$ and $\mathrm{x}$-ray fiber diffraction ${ }^{28}$ studies, respectively. Three different Poisson ratios were also tried, i.e., $\sigma=0,0.5$, and $\infty$. The first two were the extreme values expected for a homogeneous elastic rod, ${ }^{41}$ whereas the final value $\sigma=\infty$ corresponded to the (unphysical) situation where bending motions were suppressed. The torsional rigidity of calf thymus DNA obtained from the present analysis of $C=1.43 \times 10^{-19} \mathrm{erg} \mathrm{cm}$, with $\sigma=0.5$ and $\epsilon=70^{\circ}$, should be compared with the value of 1.17 $\times 10^{-19} \mathrm{erg} \mathrm{cm}$ obtained with $\sigma=\infty$ and $\epsilon=90^{\circ}$, as was assumed in our previous work. ${ }^{7}$ The relatively small difference between these two values was due to a partial cancellation of the errors produced by ignoring the bending motions and by assuming that the ethidium transition dipole was perpendicular to the helix axis. Including the dye tilt but ignoring the bending motions, as was done by 
Thomas et $a l,,^{8}$ produced an error of almost $50 \%$ in the torsional rigidity (Table III). These calculations demonstrate the importance of having accurate values for the helix parameters $\epsilon$ and $\sigma$ (or $E I$ ) if accurate, absolute torsional rigidities are to be obtained from fluorescence depolarization experiments.

\section{Synthetic polynucleotides}

The results of the fluorescence depolarization experiments on the synthetic polynucleotides $(100 \mu \mathrm{g} / \mathrm{ml}$ in 0.1 M Tris $\cdot \mathrm{HCl}, p \mathrm{H} 7.7 / 0.15 \mathrm{M} \mathrm{NaCl} ; P / D \simeq 120$ ) are summarized in Table IV. Preliminary results for some of the polynucleotides were presented in a previous communication to this journal. ${ }^{15}$ For the present work, these results were refined by a more complete analysis using the procedure outlined in Sec. IV A, and the results for two new polynucleotides poly $(d A) \cdot \operatorname{poly}(d T)$ and poly $(d G) \cdot \operatorname{poly}(d C)$ were added. The results for native and denatured calf thymus DNA and the supercoiled plasmid DNA are also included in Table $\mathrm{T}$ for comparison.

Our results revealed a number of differences among the polynucleotides, which can be summarized as follows: (1) The alternating copolymers polyd $(\mathrm{G}-\mathrm{C})^{-}$ - poly $d(\mathrm{G}-\mathrm{C})$ and poly $d(\mathrm{~A}-\mathrm{T}) \cdot$ poly $d(\mathrm{~A}-\mathrm{T})$ had quite different torsional rigidities than the homopolymers with the same base composition poly $(d \mathrm{G}) \cdot \operatorname{poly}(d \mathrm{C})$ and $\operatorname{poly}(d \mathrm{~A}) \cdot \operatorname{poly}(d \mathrm{~T})$. (2) The torsional rigidities of $\mathrm{A}-\mathrm{T}$ and $\mathrm{G}-\mathrm{C}$ containing polynucleotides were very dif ferent in the alternating copolymers, but quite similar in the homopolymers. (3) The polyribonucleotide poly $(r \mathrm{~A}) \cdot \operatorname{poly}(r \mathrm{U})$ had a significantly larger torsional rigidity $\left(C=1.53 \times 10^{-19} \mathrm{erg} \mathrm{cm}\right)$ than its deoxy counter part poly $(d A) \cdot \operatorname{poly}(d \mathrm{U})\left(C=0.83 \times 10^{-19} \mathrm{erg} \mathrm{cm}\right)$. Furthermore, the torsional rigidity of $\operatorname{poly}(d A) \cdot \operatorname{poly}(d \mathrm{U})$ increased to $1.1 \times 10^{-19}$ erg $\mathrm{cm}$ upon raising the salt concentration to $1 \mathrm{M}$, whereas the torsional rigidity of poly $(r \mathrm{~A}) \cdot \operatorname{poly}(r \mathrm{U})$ showed no change. (4) The torsional rigidity of the triple helical poly $(d \mathrm{~A}) \cdot \operatorname{poly}(r \mathrm{U}) \cdot \operatorname{poly}(r \mathrm{U})$ was considerably greater than that of any of the double helical polynucleotides. (5) The dye-wobble angle was

TABLE IV. Torsional rigidities of the polynucleotides.

\begin{tabular}{lll}
\hline Sample & $c\left(10^{-19} \mathrm{erg} \mathrm{cm}\right)$ & $\Xi^{1 / 2}(\mathrm{deg})$ \\
\hline $\mathrm{CT}$ & $1.43(0.11)^{2}$ & $17(2)$ \\
$d(\mathrm{G}-\mathrm{C}) \cdot d(\mathrm{G}-\mathrm{C})$ & $1.50(0.15)$ & $19(2)$ \\
$d(\mathrm{~A}-\mathrm{T}) \cdot d(\mathrm{~A}-\mathrm{T})$ & $0.90(0.22)$ & $12(2)$ \\
$d G \cdot d \mathrm{C}$ & $1.19(0.10)$ & $10(2)$ \\
$d \mathrm{~A} \cdot d \mathrm{~T}$ & $1.27(0.17)$ & $12(7)$ \\
$d \mathrm{~A} \cdot d \mathrm{U}$ & $0.83(0.12)$ & $15(2)$ \\
$r \mathrm{~A} \cdot \mathrm{rU}$ & $1.53(0.13)$ & $6(4)$ \\
$d \mathrm{~A} \cdot r \mathrm{U} \cdot r \mathrm{U}$ & $2.65(0.33)$ & $15(5)$ \\
$\mathrm{pBR} 322$ & $1.95(0.18)$ & $20(2)$ \\
Denatured $\mathrm{CT}$ & $0.90(0.10)$ & $0(2)$ \\
\hline$=$
\end{tabular}

${ }^{a}$ Values in parentheses are $95 \%$ confidence intervals.

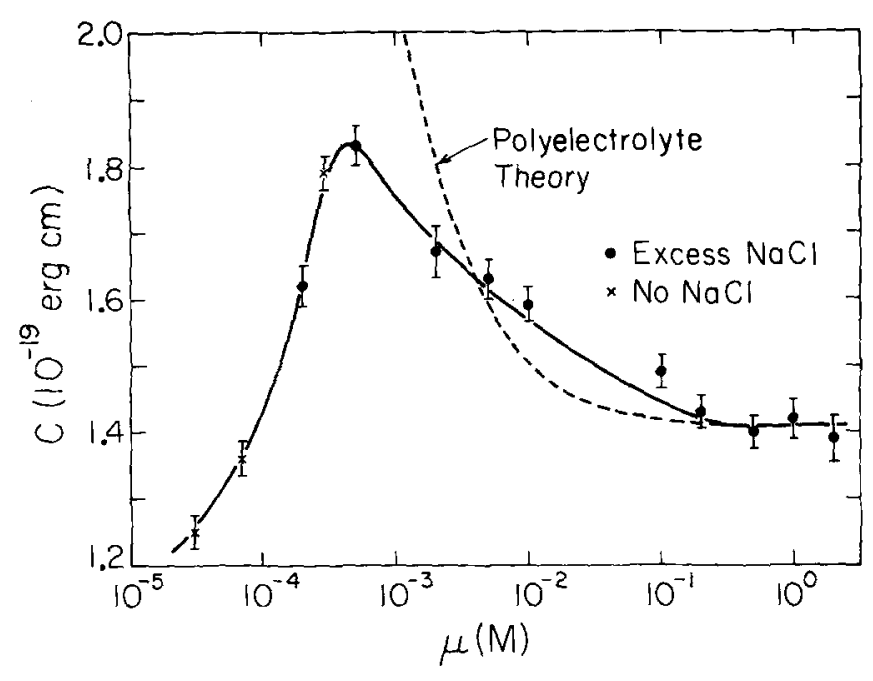

FIG. 4. Dependence of the torsional rigidity of calf thymus DNA on ionic strength $\mu$. Error bars are one standard deviation. Results are shown for solutions containing no added $\mathrm{NaCl}$ (crosses) and containing excess $\mathrm{NaCl}$ (dots). The smooth line is shown to connect the points. The broken line was calculated from Eqs. (20) and (21) together with the equations for the persistence length discussed in Sec. IV $F$ of the text.

relatively large for all polydeoxy ribonucleotides $\left(10^{\circ}-\right.$ $19^{\circ}$ ) but very small for the polyribonucleotide poly $(r \mathrm{~A})$ - $\operatorname{poly}(r \mathrm{U})$, about $6^{\circ}$. There was no apparent correlation between the dye-wobble angle and either the torsional rigidity of the helix, the fluorescence lifetime of the intercalated dye, or the fraction of nonintercalated dye.

\section{E. Polyelectrolyte effects}

Figure 4 shows a plot of the measured torsional rigidity of calf thymus DNA as a function of ionic strength $\mu$ covering the range $3 \times 10^{-5}-2.0 \mathrm{M}$. Most of the samples contained at least a tenfold excess of added $1: 1$ electrolyte $(\mathrm{NaCl})$. For those few samples in which there was no added electrolyte, the ionic strength was taken to be equal to the nucleotide concentration. From this plot (Fig, 4) several regions were distingishable. In region I, which covered the ionic strength range $0.2 \leq \mu \leq 2.0 \mathrm{M}$, the torsional rigidity was independent of ionic strength with a mean value of about 1.4 $\times 10^{-19} \mathrm{erg} \mathrm{cm}$. In region II $\left(5 \times 10^{-4} \lesssim \mu \lesssim 0.1 \mathrm{M}\right)$, the torsional rigidity increased monotonically with decreasing ionic strength, reaching a maximal value of about 1.8 $\times 10^{-19} \mathrm{erg} \mathrm{cm}$. Finally, at ionic strengths less than $5 \times 10^{-4} \mathrm{M}$ (region III), the torsional rigidity fell rapidly.

\section{DISCUSSION}

\section{A. The bound species}

The isotropic $\left(54.7^{\circ}\right)$ fluorescence decays of all the nucleic acids exhibited a fast and slow component, which we have attributed to nonintercalated and intercalated dye species, respectively. Olmsted and Kearns ${ }^{44}$ have shown that the much longer lifetime of the intercalated dye compared with free ethidium bromide is due to a reduction of the rate of an excited state proton transfer reaction with polar solvents. Variations in the lifetime 
of the intercalated dye are presumably due to different degrees of exposure of the dye to solvent, due to differences in double-helical structure. This mechanism is probably responsible for the consistently shorter lifetime of ethidium intercalated in homopolymers compared with alternating copolymers, and for the lifetime differences between polydeoxyribonucleotides and polyribonucleotides (Table I). The observed correlation between longer lifetimes of the intercalated dye and the lower fractions of nonintercalated dye is also consistent with this mechanism; the more strongly intercalated dye is the least exposed to solvent.

Equilibrium and kinetic studies ${ }^{23}$ of the interaction between ethidium bromide and DNA have revealed the presence of three bound dye species, an electrostatically bound outside form $\mathrm{ED}_{0}$ which accounts for $\sim 8 \%$ of the total bound dye at $1 \mathrm{M} \mathrm{NaCl}$, and two intercalated species $\operatorname{ED}_{1}(\sim 12 \%)$ and $\operatorname{ED}_{2}(\sim 80 \%)$. Recent studies ${ }^{45}$ with a range of intercalators have shown that $E D_{1}$ and $E D_{2}$ correspond to intercalation at sites in the major and minor grooves of the DNA double helix, respectively. $E D_{1}$ and $E D_{2}$ are thus expected to have very similar lifetimes, much longer than for unbound dye, whereas $E D_{0}$ is expected to have a lifetime intermediate between the intercalated species and the unbound dye. The lifetime $\tau_{f}$ and fraction $f$ of the fast component are in good agreement with this hypothesis ( $f=12 \%$ at 1 $\mathrm{M} \mathrm{NaCl}$ ), and we therefore attribute this component to the outside form $\mathrm{ED}_{0}$, with a possible contribution from unbound dye. The long component is attributed to the combination of $\mathrm{ED}_{1}$ and $\mathrm{ED}_{2}$. The excellent agreement between the fluorescence anisotropy decay and the elastic model implies that $E D_{1}$ and $E D_{2}$ have very similar tilt angles $\epsilon$ relative to the helix axis, which is reasonable for intercalation at sites in the major and minor grooves of the helix.

\section{B. Torsional rigidity of calf thymus DNA}

The excellent agreement between $\mathrm{Eg}$. (2) and the observed fluorescence anisotropy decay demonstrates that the elastic model of Barkley and Zimm ${ }^{11}$ for both torsion and bending is valid, and therefore may be used with confidence to extract the torsional rigidity from fluorescence depolarization data. The anisotropy decay can also be well fit by the elastic model for torsion only, as done in our earlier work. ${ }^{7,15}$ The inclusion of the bending dynamics improves the fit only slightly but results in a significantly larger value for the torsional rigidity $\left(0.93 \times 10^{-19}\right.$ to $\left.1.43 \times 10^{-19} \mathrm{erg} \mathrm{cm}\right)$. Thus, it is important to include the bending contribution in order to determine accurately the torsional rigidity. A similar situation is encountered when attempting to determine the torsional rigidity from supercoil linking number distributions ${ }^{12,48,49}$ (see Sec. V C).

The dependence of the torsional rigidity on other helix parameters is an unfortunate limitation of this technique (see Table III). However, we have shown that the helix radius and Poisson ratio may be independently determined from published hydrodynamic data, leaving the dye-tilt angle as the only unknown parameter. Fortunately, this was recently measured for native DNA's by electric dichroism. ${ }^{19}$ Thus, at least for calf thymus DNA, the torsional rigidity has been extracted with considerable certainty. Our value for calf thymus DNA under standard conditions $\left[C=(1.43 \pm 0.11) \times 10^{-19} \mathrm{erg} \mathrm{cm}\right]$ should be the most accurate value attainable from fluorescence depolarization data.

Another quantity of interest is the rms average fluctuation in the torsion angle between adjacent base pairs ${ }^{11}$ $\langle\Delta \gamma\rangle_{\text {rms }}=\left(h k_{B} T / C\right)^{1 / 2}$, where $h=3.4 \AA$ is the distance between base pairs. For the value of $C$ above, we calculate $\langle\Delta \gamma\rangle_{\mathrm{r}}=5.7^{\circ}$. The corresponding quantity for bending $\langle\Delta \alpha\rangle_{\text {rm }}$ is obtained by replacing $C$ with the bending rigidity $E I$; the result is $\langle\Delta \alpha\rangle_{\mathrm{rms}}=4.6^{\circ}$. It is clear that the structure of DNA in solution undergoes considerable fluctuations; our results show that the structure of B-DNA in solution fluctuates between a ninefold and a 12-fold double helix due to thermal motion.

\section{Other work}

Thomas et al. ${ }^{8}$ have recently reported time-dependent fluorescence depolarization measurements on ethidium intercalated in viral $\phi 29$ DNA at $0.01 \mathrm{M} \mathrm{NaCl}$. They concluded that the intermediate decay zone of the bead-spring model, ${ }^{46}$ which is equivalent ${ }^{11}$ to the elastic model discussed in Sec. II without the bending dynamics, provided the best fit to their data, with a torsional rigidity $C=1.29 \times 10^{-19} \mathrm{erg} \mathrm{cm}$. This value agreed well with the torsional rigidity of calf thymus DNA reported by us ${ }^{7}$ $\left(C=1.3 \times 10^{-19} \mathrm{erg} \mathrm{cm}\right)$. However, this agreement is somewhat fortuitous, since the data were obtained under different ionic strength conditions, and a different dye-tilt angle and helix radius were used in the analysis. The results in Sec. IV E clearly show that there is an $\sim 12 \%$ polyelectrolyte contribution to the torsional rigidity at $0.01 \mathrm{M} \mathrm{NaCl}$. The apparent agreement between our value, at $0.15 \mathrm{M} \mathrm{NaCl}$, and that of Thomas et al. ${ }^{8}$ at $0.01 \mathrm{M} \mathrm{NaCl}$, results from a cancellation between the effects of different radius and dye tilt used in the analysis, and the difference in polyelectrolyte contribution between 0.01 and $0.15 \mathrm{M}$. However, our data at 0.01 $\mathrm{M} \mathrm{NaCl}$ (Sec. IV E) do agree with Thomas et al. ${ }^{8}$ if analyzed in the same way $\left(\epsilon=70^{\circ}, b=12 \AA\right.$, and $\left.\sigma=\infty\right)$. There is thus no difference in the intrinsic torsional rigidities of calf thymus DNA and viral $\phi 29$ DNA. The refined analysis of the fluorescence depolarization data presented here is more complete than that of Thomas et al. ${ }^{8}$ in that we have included the bending dynamics and used a helix radius and Poisson ratio determined from independent hydrodynamic data.

Recently, Hurley et al. ${ }^{14,47}$ have used EPR spectroscopy of intercalated spin probes to study the torsional dynamics of DNA. On the basis of the observed dependence of the rotational correlation time on the length of the DNA, it was concluded that the dynamics were consistent with a bead-spring model, essentially equivalent to the elastic model of Barkely and $\mathrm{Zimm}^{11}$ (Sec. II) except that bending was ignored. Using this model, the rms average amplitude of the torsional fluctuations of a single base pair could be related to the rotational correlation time. The torsional rigidity was then calculated from the rms amplitude. This method for mea- 
suring the torsional rigidity is thus indirect, depending on a single rotational correlation time inferred from the EPR spectrum, in contrast to our method in which the time dependence of the entire rotational correlation function is fit to the elastic model by least-squares adjustment of the torsional rigidity. Nevertheless, the rigidity obtained by Hurley et al. for DNA intercalated by an ethidium spin probe $\left(C=1.2 \times 10^{-19} \mathrm{erg} \mathrm{cm}\right)^{14,47}$ is in fair agreement with our value $\left(C=1.43 \times 10^{-19}\right.$ erg $\mathrm{cm}$ ). The difference is probably due to the neglect of the bending motions in the analysis of the EPR data.

Estimates of the torsional rigidity of DNA have in the past been derived from the equilibrium linking number distributions of closed circular supercoiled DNA after enzymatic conversion from the nicked to the closed form. ${ }^{48,49}$ For example, Depew and Wang ${ }^{48}$ estimate that $C$ is in the range $(0.64-1.1) \times 10^{-19} \mathrm{erg} \mathrm{cm}$, which is a lower limit since the bending energy was ignored in their calculation. More recently, Vologodskii et al. ${ }^{12}$ have made an exact calculation of the bending contribution to the linking number distribution using statistical methods. They found that the width of the linking number distribution had approximately equal contributions from twisting and bending. The torsional rigidity calculated from the width of the twisting distribution was $C=(1.65$ $\pm 0.33) \times 10^{-19} \mathrm{erg} \mathrm{cm}$. This result depends on the value they chose for the bending rigidity (corresponding to a persistence length of $575 \AA)^{12}$ but is in good agreement with our value of $C=1.43 \times 10^{-19} \mathrm{erg} \mathrm{cm}$ (obtained for a persistence length of $532 \AA$ ), and is a better estimate than was originally inferred from the supercoiling data. ${ }^{48,49}$

Theoretical simulations of DNA structure, ${ }^{43,50}$ wherein the total helix energy can be calculated as a function of the local twist angle, also provide estimates of the torsional rigidity. From Levitt's calculations ${ }^{43}$ for a 20 base pair segment of straight DNA, a torsional rigidity $C=20 \times 10^{-19}$ erg cm was calculated, ${ }^{14}$ while Miller's calculations ${ }^{50}$ for a $d(\mathrm{G}-\mathrm{C}) \cdot d(\mathrm{C}-\mathrm{G})$ dinucleotide implied $C=36 \times 10^{-19} \mathrm{erg} \mathrm{cm} .14$ Both theoretical calculations overestimate the torsional rigidity by more than an order of magnitude, presumably due to the excessive constraints imposed on the model structures, ${ }^{43,50}$ or due to the omission of helix-solvent interactions in the calculation.

Recent NMR studies of small fragments of double helical DNA have indicated the presence of large amplitude $\left( \pm 20^{\circ}-35^{\circ}\right)$ conformational fluctuations of the phosphodiester backbone, the deoxyribose sugar, and the base planes with time constants near 1 ns. ${ }^{57-59} \mathrm{~A}$ coresponding large amplitude relaxation with a 1 ns time constant was not observed in the fluorescence depolarization of calf thymus DNA or any of the synthetic polynucleotides. However, a more recent NMR study of native high molecular weight $\mathrm{DNA}^{60}$ found no evidence for a 1 ns internal motion, the observed microsecond correlation times being consistent with the long-range bending flexibility of DNA. In view of the discrepancy in the NMR work, it is not clear whether a 1 ns internal motion should be observable in the fluorescence depolarization of our DNA samples. These internal motions may, in fact, be unique to short rodlike DNA molecules only.

\section{Structural effects}

The largest torsional rigidity was observed for the triple helix ${ }^{29}$ poly $(d A) \cdot \operatorname{poly}(r U) \cdot \operatorname{poly}(r U)$. Since the tor sional rigidity depends on the helix radius, according to $C=\frac{1}{2} \mu \pi b^{4}$ (for a uniform cylinder of radius $b$ and torsional modulus $\mu$ ), ${ }^{51}$ a larger rigidity is expected for the triple helix. Using $b=14.8 \AA$ for the triple helix and $b=13.4 \AA$ for the double helix, we calculate $C($ triple $) / C$ (double $)=1.3$, compared with the observed value of 1.9. Thus, the torsional modulus of the triple helix is larger than the double helix $\mu$ (triple) $/ \mu$ (double) $=1.5$, indicating that the triple helix is inherently less flexible. Since the triple helix can be considered as a $\operatorname{poly}(d A) \cdot \operatorname{poly}(r U)$ double helix with an extra poly $(r U)$ strand accommodated in the major groove, ${ }^{29}$ the implication is that binding large molecules to the DNA double helix reduces its conformational mobility.

The alternating self-complementary copolymer poly $d(\mathrm{~A}-\mathrm{T}) \cdot$ poly $d(\mathrm{~A}-\mathrm{T})$ is considerably more flexible than native $B-D N A$, as indicated by its smaller torsional rigidity $\left(C=0.90 \times 10^{-19} \mathrm{erg} \mathrm{cm}\right)$. Several physical experiments have shown that poly $d(\mathrm{~A}-\mathrm{T}) \cdot$ poly $d(\mathrm{~A}-\mathrm{T})$ forms hairpin helix branches without undergoing melting. ${ }^{52}$ Hairpin branches are formed when a single strand in an open region of the DNA (not base paired) closes on itself into a double helix, which is possible because of the self-complementary base sequence. The ability of the DNA to open without melting confers an enhanced conformational mobility that has been observed, even at room temperature, in viscosity measurements and in the unusual activity of poly $d(A-T) \cdot$ poly $d(A-T)$ in various enzymatic reactions. ${ }^{53}$ Our results demonstrate that this enhanced conformational mobility is also manifested in the torsional rigidity.

Thermally denatured native DNA also has a much smaller torsional rigidity than intact native $B-D N A$. This suggests that the random coil form of DNA is more flexible than the double helix, and therefore that the torsional rigidity of DNA is determined by the relative fractions of double-helical and random coil regions. It is interesting that poly $(d \mathrm{~A}) \cdot \operatorname{poly}(d \mathrm{U})$ had a small torsional rigidity at $0.15 \mathrm{M} \mathrm{NaCl}$, which actually increased towards the rigidity of $\operatorname{poly}(d \mathrm{~A}) \cdot \operatorname{poly}(d \mathrm{~T})$ and poly $(d \mathrm{G})$ - poly $(d \mathrm{C})$ upon increasing ionic strength to $1 \mathrm{M}$. This unusual behavior is consistent with a significant fraction of unpaired bases at $0.15 \mathrm{M} \mathrm{NaCl}$. Thus, our results imply that poly $(d A) \cdot \operatorname{poly}(d U)$ is a particularly unstable homopolymer. By contrast, poly $(r A) \cdot \operatorname{poly}(r U)$ was considerably more rigid and did not show this salt effect [the torsional rigidity of poly $(r A) \cdot \operatorname{poly}(r \mathrm{U})$ in the high salt limit was the same as shown in Fig. 4].

The presence of hairpin helix branches and singlestranded regions in the DNA means that the structure is not uniform and that the elastic continuum model is therefore not strictly valid. However, a structure will be effectively uniform if the wavelengths of the torsional modes are much longer than the scale of the inhomogeneities. The average wavelength is about 100 base 
TABLE V. Sequence dependent properties.

\begin{tabular}{|c|c|c|c|}
\hline Sequence & $C\left(10^{-19}\right.$ erg $\left.\mathrm{cm}\right)$ & $T_{m}\left({ }^{\circ} \mathrm{C}\right)^{\mathrm{a}}$ & $E_{s}\left(\mathrm{kcal} \mathrm{mol} l^{-1}\right)^{\mathrm{b}}$ \\
\hline$d(\mathrm{G}-\mathrm{C}) \cdot d(\mathrm{G}-\mathrm{C})$ & 1.50 & 115 & -11.3 \\
\hline$d \mathrm{~A} \cdot d \mathbf{T}$ & 1.27 & 68.5 & -6.3 \\
\hline$d \mathrm{G} \cdot d \mathrm{C}$ & 1.19 & 101 & -5.2 \\
\hline$d(\mathrm{~A}-\mathrm{T}) \cdot d(\mathrm{~A}-\mathrm{T})$ & 0.90 & 61 & -5.0 \\
\hline
\end{tabular}

${ }^{2}$ Reference 61 .

${ }^{b}$ Calculated values reported in Ref. 62. Very similar values are obtained by averaging the results of the five theoretical calculations reviewed in Ref. 63.

pair separations [calculated from Eq. (5) for a $25 \mathrm{~ns}$ average relaxation time]. Inhomogeneities shorter than this will have no observable effect. The depolarization data for both poly $d(\mathrm{~A}-\mathrm{T}) \cdot$ poly $d(\mathrm{~A}-\mathrm{T})$ and partially denatured calf thymus DNA fit the model very well (1.1 $\left.\lesssim \chi_{r}^{2} \lesssim 1.3\right)$, indicating that the nonduplex regions in these samples are sufficiently short, i. $e_{.}$, much shorter than 100 base pairs. However, poly $(d \mathrm{~A}) \cdot \operatorname{poly}(d \mathrm{U})$ did show some deviation from the model $\left(\chi_{r}^{2} \sim 4\right)$, consistent with the idea that this sample was more denatured.

All of the nucleic acids discussed so far have simple tertiary structures; they are linear double helices. Our plasmid DNA sample ( $\mathrm{pBR} 322$ ), however, is a closed circular DNA with superhelical tertiary turns. The anisotropy decay of ethidium intercalated in this DNA agreed well with Eq. (2), but decayed more slowly than for native $B-D N A$; the torsional rigidity was $C=1.95$ $\times 10^{-19}$ erg cm, assuming that $b=13.4 \AA$. Supercoiled DNA is thus more rigid than linear DNA, which may result from the torsional stress imposed on the double helix due to supercoiling. ${ }^{54}$ A dependence of the torsional rigidity on the imposed torsional stress implies the existence of nonlinear terms in the torsional restoring force. Although our result for one supercoiled DNA hardly constitutes a proof of this nonlinearity, it would be possible to test this hypothesis by anisotropy measurements on a series of closed circular DNA's with varying numbers of superhelical turns. It should also be possible to test the prediction ${ }^{54}$ that sufficiently supercoiled DNA will locally denature to relax the imposed stresses, since we have found that the torsional rigidity is sensitive to local denaturation.

It is possible that the slower anisotropy decay of $\mathrm{pBR}$ 322 DNA is due to an effectively larger helix radius that may result from a tertiary conformation in which the DNA is densely interwound. It is known that supercoiled DNA adopts several tertiary conformations in solution, depending on the ionic strength ${ }^{55}$ and number of superhelical turns. ${ }^{56}$ Since the tertiary conformation of our sample is unknown, we are unable to eliminate this possiblity. However, whether the effect is due to the imposed torsional stresses or the tertiary conformation, it is clear that the anisotropy decay is sensitive to the tertiary structure of DNA.

Our study of the synthetic polynucleotide complexes and supercoiled plasmid DNA has revealed that the torsional rigidity is sensitive to differences in nucleic acid structure. The major effect is due to helical structure; our results demonstrate that the magnitude of the torsional rigidity of double-stranded complexes reflects the extent of helical structure, since the intrinsic rigidities of the double helix and the random coil are quite different. The intrinsic rigidity of a triple helix is a factor of 2 larger than that of the double helix. The second important structural effect is due to tertiary structure, since we have found that the torsional rigidity is increased by supercoiling.

In view of these findings, the possibility of employing the anisotropy decay as a probe of these structural features should be considered. We have already mentioned using torsional rigidity measurements to monitor local denaturation in over stressed supercoiled DNA. Other applications should also be possible.

\section{E. Sequence effects}

We now consider the differences in torsional rigidity among the intact double helical polydeoxyribo nucleotides in Table IV. The most striking difference is between the homopolymers and the alternating copolymers; for example, poly $d(\mathrm{G}-\mathrm{C}) \cdot$ poly $d(\mathrm{G}-\mathrm{C})$ is considerably more rigid than poly $(d G) \cdot \operatorname{poly}(d C)$. These results suggest the possibility of a sequence-dependent contribution to the torsional rigidity, which could arise from basebase interactions. It is obvious that this contribution cannot be due to base pairing interactions alone.

In Table $\mathrm{V}$ we attempt to correlate the torsional rigidity with two sequence dependent properties of the polynucleotides that are sensitive to base-base interactions: the melting temperature $T_{m},{ }^{81}$ which measures the overall thermodynamic stability of the doublestranded polynucleotide complex, and the calculated base-stacking interaction energies, ${ }^{62}$ which measure the electronic interactions between adjacent bases. The melting temperature has contributions from both base pairing and base stacking. These values were taken from the literature. ${ }^{61,62}$ Although there is some variation in calculated stacking energies reported in the literature, the order of the stacking energies shown in Table Vi is consistent with the majority of the theoretical calculations. These values are also representative of the several different calculations that have been reviewed recently by Rein. ${ }^{63}$

It is significant that the thermodynamically most stable 
[poly $d(\mathrm{G}-\mathrm{C}) \cdot$ poly $d(\mathrm{G}-\mathrm{C})$ ] and the least stable [poly $d(\mathrm{~A}-$ $\mathrm{T}) \cdot$ poly $d(\mathrm{~A}-\mathrm{T})]$ are also the most rigid and the least rigid, respectively. However, the correlation of $C$ with $T_{m}$ breaks down for poly $(d \mathrm{~A}) \cdot \operatorname{poly}(d \mathrm{~T})$, which has a smaller $T_{m}$ than poly $(d \mathrm{G}) \cdot \operatorname{poly}(d \mathrm{C})$ but is more rigid. The torsional rigidities of all the polynucleotides do, however, correlate with the calculated stacking energies; the order is preserved and the magnitude of the rigidity differences is reflected in the differences in stacking energy, except for poly $d(\mathrm{~A}-\mathrm{T}) \cdot$ poly $d(\mathrm{~A}-\mathrm{T})$ which has an unexpectedly small torsional rigidity.

Why should the torsional rigidity be partly determined by base-stacking interactions? Recall that the torsional rigidities we have measured imply considerable relative motion between adjacent base pairs, as indicated by $\langle\Delta \gamma\rangle_{\mathrm{rms}}=5.7^{\circ}$. It is reasonable that strong attractive interactions between adjacent base pairs will influence their relative motion. It is also reasonable that these interactions will depend on the torsion angle: a large overwinding or underwinding of the double helix will disrupt the stacking interactions and so raise the helix energy.

The observed sequence effects strongly imply that base-base interactions contribute to the torsional rigidity. It is clear that base pairing interactions cannot account for these effects. While the correlation with the stacking energies is only suggestive, and of course depends on the calculated values, ${ }^{62,63}$ it is reasonable that the sequence effects could arise from base-stacking interactions.

\section{F. Polyelectrolyte effects}

Currently, there is considerable interest in the polyelectrolyte contribution to the stiffness of DNA. Previous experimental ${ }^{40,64,65}$ and theoretical work $\mathrm{k}^{24,66,69}$ has dealt solely with the bending rigidity or the persistence length of native DNA. Our results, presented in Fig. 4 , constitute the first measurements of the polyelectrolyte contribution to the torsional rigidity. Although a quantitative understanding of these results is not yet possible, due to the lack of a theory for the change in torsional rigidity with ionic strength, the results are consistent with the following qualitative arguments.

At ionic strengths greater than about $0.2 \mathrm{M}$ (region I), the Debye-Hückel screening length $\kappa^{-1}$ is less than the effective contour distance between charges on the DNA phosphate backbone after counterion condensation $\left(\sim 7.14 \AA\right.$, the Bjerrum length, in water at $\left.25^{\circ} \mathrm{C}\right)$. Therefore, the charges are effectively screened from one another and there should be no significant polyelectrolyte contribution to the torsional rigidity (or the bending rigidity). The torsional rigidity is thus expected to be independent of ionic strength in this region, as was observed experimentally (Fig. 4). Since our measurements extend only to $2 \mathrm{M} \mathrm{NaCl}$ solutions, we cannot rule out the possibility that some structural change occurs in DNA at higher salt concentrations.

At ionic strengths less than about $0.1 \mathrm{M}$ (regions II and III, the Debye-HUckel screening length is greater than the effective contour distance between charges.
Therefore, the charges begin to interact with each other and an expansion and stiffening of the chain is expected; this is the so-called polyelectrolyte contribution to the chain stiffness. Experimentally, we did in fact observe a modest increase in the torsional rigidity over the range $5 \times 10^{-4} \leq \mu \leq 0,1 \mathrm{M}$ (region II). However, at ionic strengths less than $5 \times 10^{-4} \mathrm{M}$, the torsional rigidity fell rapidly. We believe that this is due to denaturation or "melting" of the double helix caused by the electrostatic repulsions which cannot be accommodated by further expansion and elastic deformations of the chain.

A theory for the change in torsional rigidity with ionic strength is lacking at present. However, several authors have developed expressions describing the polyelectrolyte contribution to the persistence length of DNA. ${ }^{24,66,67,69}$ This work has been reviewed by Schurr and Allison, ${ }^{68}$ who have also corrected a subtle geometric error in the theory of Manning. ${ }^{24}$ According to these theories, the total persistence length is given by $P_{0}+P_{e}$, where $P_{0}$ is the intrinsic persistence length of the (uncharged) double helix and $P_{e}$ is the polyelectrolyte contribution, which can be written in the form ${ }^{69}$

$$
P_{e}=1 / 4 Q \kappa^{2} \text {, }
$$

where $Q$ is the Bjerrum length and $\kappa^{-1}$ is the DebyeHückel screening length $\kappa^{2}=8 \pi Q \mu$.

In principle, these expressions for the persistence length can be combined with Eqs. (20) and (21) to give an expression for the change in torsional rigidity with ionic strength. The broken line in Fig. 4 was calculated in this manner, assuming that the Poisson ratio $\sigma=0.5$ applies to both the intrinsic and polyelectrolyte contributions to the rigidity. The predicted change in the torsional rigidity is on the same order of magnitude as that observed experimentally for ionic strengths greater than about $10^{-3} \mathrm{M}$, but the quantitative agreement is poor. Nevertheless, both our results and the theoretical calculations ${ }^{68}$ suggest only a modest $(6 \%-$ $12 \%$ ) increase in torsional rigidity at a salt concentration of $0.01 \mathrm{M}$ compared with the $260 \%$ increase in persistence length reported by Harrington ${ }^{64}$ from flowbirefringence measurements.

The rapid drop in the torsional rigidity at ionic strengths less than $5 \times 10^{-4} \mathrm{M}$ is not predicted by the polyelectrolyte theories, which take no account of the possibility of melting of the double helix. The melting temperature $T_{m}$ as a function of ionic strength can be represented, at least approximately, by the empirical equation ${ }^{70}$

$$
T_{m}=16.6 \log _{10} \mu+41 \chi_{\mathrm{GC}}+81.5,
$$

where $\chi_{\mathrm{GC}}$ is the fractional $\mathrm{G}-\mathrm{C}$ content of the DNA. For calf thymus DNA $\left(\chi_{G C}=0.42\right)$ we calculate that the melting will occur at room temperature $\left(23^{\circ} \mathrm{C}\right)$ at an ionic strength of $3 \times 10^{-5} \mathrm{M}$. The approximate agreement between this calculated ionic strength and our experimental data (Fig. 4) further supports the idea that the drop in rigidity in region III is due to partial denaturation of the helix.

\section{G. Initial anisotropy loss}

All the nucleic acid samples studied exhibited a nonzero wobble angle $\Xi^{1 / 2}$, indicating that there was an 
initial loss of fluorescence anisotropy. In our analysis we have modeled this anisotropy loss as resulting from infinitely rapid reorientation of the ethidium transition moment in the plane of the phenanthridinium ring. This approach is justified in the case of aqueous solvent, where the reorientation was very rapid and unresolved by our photon-counting instrument. However, in high viscosity solvents, such as saturated sucrose, the reorientation is much slower, on the order of a few nanoseconds, and was time resolved. ${ }^{7}$ In a series of sucrose solutions of different viscosity, Genest and Wahl ${ }^{73}$ found that the initial anisotropy decay was approximately exponential with a decay time proportional to the viscosity. Thus, the initial rapid reorientation of the intercalated ethidium is coupled to solvent bulk viscosity. These workers also found that the reorientation amplitude increased with increasing temperature ${ }^{73}$ from which they inferred that the motion was hindered by potential barriers resulting from the deformation of the DNA-dye complex. Our present results show that the amplitude is about $15^{\circ}$ in all of the DNA samples, is smaller in RNA, about $6^{\circ}$, and is zero in thermally denatured DNA.

There are at least three possible explanations for the ini tial anisotropy loss: (1) reorientation of the nonintercalated dye (outside bound and possibly also unbound dye); (2) independent motion of the dye in its intercalation site; and (3) an extremely rapid internal motion of the DNAdye complex. We now consider each of these possibilities.

Equation (2) for the anisotropy decay applies only to the intercalated dye. A more complete expression, including the short-lived "free" component of the fluorescence, is

$$
r(t)=\frac{f \exp \left(-t / \tau_{f}\right) r_{f}(t)+(1-f) \exp \left(-t / \tau_{b}\right) r_{b}(t)}{f \exp \left(-t / \tau_{f}\right)+(1-f) \exp \left(-t / \tau_{b}\right)},
$$

where $r_{f}(t)$ is the anisotropy decay of the free component, $r_{b}(t)$ is the anisotropy decay given by Eq. (2), and the other symbols have the same meaning as in Sec. III C. Two points are apparent from this equation: that the anisotropy loss due to the free component is proportional to the fraction $f$, and that at times longer than about $3 \tau_{f}$ the anisotropy decay simplifies to that of the intercalated dye only $r_{b}(t)$. Our results show no correlation between the wobble angle $\Xi^{1 / 2}$ and the fraction $f$ of nonintercalated dye, even though the fraction was varied over a wide range $(2 \%$ to $35 \%)$ by changes in ionic strength, DNA concentration, and the particular polynucleotide to which the dye was bound. Also, the theoretical fits usually began $5 \mathrm{~ns}$ after the peak of the decay curves $\left(\approx 3 \tau_{f}\right)$. Therefore, we are confident that the initial anisotropy loss measured by $\Xi^{1 / 2}$ is not due to reorientation of the free dye, and that $\Xi^{1 / 2}$ is actually a property of the intercalated dye.

Independent motion of the dye within its intercalation site could be coupled to solvent viscosity via the frictional forces exerted by solvent on the bulky phenyl group projecting into the grooves of the DNA helix, ${ }^{74}$ as suggested by Genest and Wahl. ${ }^{73}$ Independent dye motion is consistent with a temperature dependent amplitude, and a smaller amplitude in RNA: the differences in lifetime and fraction of nonintercalated dye between $\operatorname{poly}(r A) \cdot \operatorname{poly}(r U)$ and its deoxy counterpart poly $(d A)$ - poly $(d \mathrm{U})$ imply that the dye is more strongly bound to RNA than to DNA.

A rapid internal motion of the DNA-dye complex is also consistent with all the data; a local helix motion would be coupled to solvent viscosity and could also exhibit a temperature dependent amplitude. The absence of any correlation between the wobble angle and the torsional rigidity (Table IV) implies that the internal motion cannot be a torsional or bending mode. This motion is considerably faster than the $\approx 1 \mathrm{~ns}$ internal motions inferred from the NMR work on short fragments of DNA $^{57,59}$; the reorientation we observed was faster than the 250 ps instrumental response. The smaller wobble angle for RNA may indicate that this motion is more restricted in RNA than in DNA.

A detailed understanding of the different wobble angles measured in RNA and in denatured DNA must await the assignment of the initial anisotropy loss. Although we are unable to decide between independent dye motion and a rapid internal motion of the DNA-dye complex on the basis of the present data, it should be possible to resolve this question by measurements on DNA with covalently attached ethidium monoazide probes, ${ }^{75,76}$ in which the independent dye motion would presumably be minimized.

\section{H. Conclusions}

In this paper we have attempted a comprehensive study of the torsional and bending dynamics of nucleic acids by time-resolved fluorescence depolarization. Our important findings may be summarized as follows: (1) The elastic model of Barkley and $\mathrm{Zimm}^{11}$ for both torsion and bending reproduces the experimental fluorescence anisotropy very accurately. (2) The torsional rigidity of calf thymus DNA under standard conditions is $C=(1.43$ $\pm 0.11) \times 10^{-19} \mathrm{erg} \mathrm{cm}$. This refined value includes both bending and dye tilt. (3) There is a polyelectrolyte contribution to the torsional rigidity of DNA at low ionic strengths. We measured the magnitude of this contribution over a wide range of ionic strength and found that current theories for the change in persistence length with ionic strength cannot quantitatively predict this contribution. (4) Helical structure is the major structural feature affecting the torsional rigidity. The order of torsional $r$ igidities is triple strand $>$ doubled strand $>$ single strand. (5) Supercoiled DNA is more rigid than linear DNA. (6) Small sequence effects are observed in the torsional rigidity, and probably result from base-base interactions. (7) There is an initial loss of anisotropy that is either due to a rapid internal motion of the double helix or independent dye motion. This motion has a smaller amplitude in RNA than in DNA, and is absent in denatured DNA.

The fluorescence depolarization technique is now fully estabilished as a direct and accurate probe of the internal motions of nucleic acids. We are now beginning to understand the structural and environmental effects that govern these motions and so determine the conformation- 
al flexibility of DNA. It should now be possible to extend this technique to more complex systems which are of biological interest, such as chromatin.

\section{ACKNOWLEDGMENTS}

This work has benefited tremendously from collaboration with a number of enthusiastic and helpful colleagues at Caltech. Thanks are due to Dr. Michael Becker and Dr. Horace Drew for suggestions and encouragement; to Professor Peter Dervan for gifts of some synthetic polynucleotide samples; and to Dr. Richard Scheller for preparing the supercoiled plasmid DNA. We also thank Professor J. Michael Schurr of the University of Washington for helpful telephone discussions and Professor Mary Barkley of the University of Kentucky for constructive comments. Appreciation is also expressed to the National Science Foundation for partial support of this work under Grant Nos. CHE 7905683 and DMR81-05034.

${ }^{1}$ T. Tao, Biopolymers 8, 609 (1969).

2J. Yguerabide, Methods Enzymol. 26, 498 (1972).

${ }^{3}$ G. R. Fleming, J. M. Morris, and G. W. Robinson, Chem. Phys. 17, 91 (1976).

${ }^{4}$ K. G. Spears and I. E. Cramer, Chem. Phys, 30, 1 (1978).

${ }^{5} \mathrm{Ph}$. Wahl, J. Paoletti, and J.-B. Le Pecq, Proc. Natl. Acad. Sci. U.S. A. 65, 417 (1970).

${ }^{6}$ I. Munro, I. Pecht, and I. Stryer, Proc. Natl. Acad. Sci. U.S.A. 76, 56 (1979).

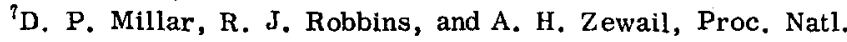
Acad. Sci. U.S. A. 77, 5593 (1980).

${ }^{8} \mathrm{~J}$. C. Thomas, S. A. Allison, C. J. Appellof, and J. M. Schurr, Biophys. Chem. 12, 177 (1980).

${ }^{9}$ M. Le Bret, Biopolymers 17, 1939 (1978).

${ }^{10} \mathrm{C}$. J. Benham, Biopolymers 18, 609 (1979).

${ }^{11}$ M. D. Barkley and B. H. Zimm, J. Chem. Phys. 70, 2991 (1979).

${ }^{12}$ A. V. Vologodskii, V. V. Anshelevich, A. V. Lukashin, and M. D. Frank-Kamenetskii, Nature (London) 280, 294 (1979).

${ }^{13} \mathrm{~S}$. W. Englander and J. J. Englander, Methods Enzymol. 49, 24 (1978)

${ }^{14}$ I. Hurley, B. H. Robinson, C. P. Scholes, and L. S. Lerman, in Nucleic Acid Geometry and Dynamics, edited by $\mathrm{R}$. H. Sarma (Pergamon, New York, 1980).

${ }^{15}$ D. P. Millar, R. J. Robbins, and A. H. Zewail, J. Chem. Phys. 74, 4200 (1981).

${ }^{16}$ T. J. Chuang and K. B. Eisenthal, J. Chem. Phys. 57, 5094 (1972).

${ }^{17}$ M. J. Waring, J. Mol. Biol. 13, 269 (1965).

${ }^{18} \mathrm{~J} .-B$. LePecq and C. Paoletti, J. Mol. Biol. 27, 87 (1967).

${ }^{19} \mathrm{M}$. Hogan, N. Dattagupta, and D. M. Crothers, Biochemistry $18,280(1979)$

${ }^{20}$ D. W. Marquardt, J. Soc. Ind. Appl. Math. 11, 431 (1963).

${ }^{21}$ P. R. Bevington, Data Reduction and Error Analysis for the Physical Sciences (McGraw-Hill, New York, 1969).

${ }^{22} \mathrm{~A}$. Grinvald and I. Z, Steinberg, Anal. Biochem. 59, 583 (1974).

${ }^{23} \mathrm{~J}$. L. Bresloff and D. M. Crothers, J. Mol. Biol. 96, 103 (1975).

${ }^{24}$ G. S. Manning, Q. Rev. Biophys. 11, 179 (1978).

${ }^{25}$ D. W. Wilson and I. G. Lopp, Biopolymers 18, 3025 (1979).

${ }^{26} \mathrm{H}$. Yamakawa and M. Fujii, Macromolecules 6, 407 (1973).

${ }^{27} \mathrm{R}$. T. Kovacic and K. E. van Holde, Biochemistry 16, 1490 (1977).
${ }^{28}$ S. Arnott, Prog. Biophys. Mol. Biol. 21, 267 (1970).

${ }^{29} \mathrm{~S}$. Arnott and P. J. Bond, Nature (London) 244, 99 (1973).

${ }^{30}$ R. H. Scheller, T. L. Thomas, A. S. Lee, W. H. Klein, W. D. Niles, R. J. Britten, and E. H. Davidson, Science 196, 197 (1977).

${ }^{31} \mathrm{~K}$. E. Reinert, J. Strassburger, and H. Triebel, Biopolymers 10, 285 (1971).

${ }^{32}$ A. Prunell and G. Bernardi, J. Biol. Chem. 218, 3433 (1973).

${ }^{33}$ M. T. Record, C. P. Woodbury, and R. B. Inman, Biopolymers 14, 393 (1975).

${ }^{34} \mathrm{~J}$. E. Godfrey, Biophys. Chem. 5, 285 (1976).

${ }^{35} \mathrm{H}$. Yamakawa and M. Fujii, Macromolecules 7, 649 (1974).

${ }^{36} \mathrm{D}$. Jolly and H. Eisenberg, Biopolymers 15, 61 (1976).

${ }^{37} \mathrm{~J}$. E. Godfrey and H. Eisenberg, Biophys. Chem. 5, 301 (1976).

${ }^{38} \mathrm{G}$. Voordouw, Z. Kam, N. Borochov, and H. Eisenberg, Biophys. Chem. 8, 171 (1978).

${ }^{39} \mathrm{H}$. Yamakawa and M. Fujii, Macromolecules 7, 128 (1974),

${ }^{40} \mathrm{C}$. Frontali, E. Dore, A. Ferrauto, E. Gratton, A. Bettini, M. R. Pozzan, and E. Valdevit, Biopolymers 18, 1353 (1979).

${ }^{41}$ L. D. Landau and E. M. Lifshitz, Theory of Elasticity (Pergamon, Oxford, 1970), 2nd edition, pp. 13-14.

${ }^{42}$ M. Hogan, N. Dattagupta, and D. M. Crothers, Proc. Natl. Acad. Sci. U.S. A. 75, 195 (1978).

${ }^{43}$ M. Levitt, Proc. Natl. Acad. Sci. U.S.A. 75, 640 (1978).

${ }^{44} \mathrm{~J}$. Olmsted III and D. R. Kearns, Biochemistry 16, 3647 (1977).

${ }^{45}$ L. P. G. Wakelin and M. J. Waring, J. Mol. Biol. 144, 183 (1980).

${ }^{46}$ S. A. Allison and J. M. Schurr, Chem. Phys. 41, 35 (1979).

${ }^{47}$ B. H. Robinson, L. S. Lerman, A. H. Beth, H. L. Frisch, L. R. Dalton, and C. Auer, J. Mol. Biol. 139, 19 (1980).

${ }^{48}$ R. E. Depew and J. C. Wang, Proc. Natl. Acad. Sci. U.S.A. 72, 4275 (1975).

${ }^{49}$ D. E. Pulleybank, M. Shure, D. Tang, J. Vinograd, and H. P. Vosberg, Proc. Natl. Acad. Sci. U.S. A. 72, 4280 (1975).

${ }^{50}$ K. J. Miller, Biopolymers 18, 959 (1979).

${ }^{51}$ L. D. Landau and E. M. Lif shitz, Theory of Elasticity (Pergamon, Oxford, 1970), 2nd edition, p. 74.

${ }^{52}$ R. L. Baldwin, Molecular Associations in Biology, edited by B. Pullman (Academic, New York, 1968), p. 145.

${ }^{53}$ R. I. Baldwin, Acc. Chem. Res. 4, 265 (1971).

${ }^{54}$ C. J. Benham, Proc. Natl. Acad. Sci. U.S.A. 76, 3870 (1979).

${ }^{55}$ A. M. Campbell, Biochem. J. 171, 281 (1978).

${ }^{56}$ A. M. Campbell and D. J. Jolly, Biochem. J. 133, 209 (1973).

${ }^{57}$ M. E. Hogan and O. Jardetzky, Biochemistry 19, 3460 (1980).

${ }^{58}$ P. H. Bolton and T. I. James, J. Am. Chem. Soc. 102, 25 (1980).

${ }^{59}$ P. H. Bolton and T. I. James, Biochemistry 19, 1388 (1980).

${ }^{60}$ S. J. Opella, W. B. Wise, and J. A. DiVerdi, Biochemistry 20, 284 (1981).

${ }^{61}$ R. D. Wells, J. E. Larson, R. C. Grant, B. E. Shortle, and C. R. Cantor, J. Mol. Biol. 54, 465 (1970).

${ }^{62}$ R. Rein, N. S. Goel, N. Fukuda, M. Pollack, and P. Claverie, Ann. N. Y. Acad. Sci. 153, 805 (1969).

${ }^{63} \mathrm{R}$. Rein, Intermolecular Interactions, edited by B. Pullman (Wiley, New York, 1978), Vol. 1, p. 307.

${ }^{64}$ R. E. Harrington, Biopolymers 17, 919 (1978).

${ }^{65}$ N. Borochov, H. Eisenberg, and Z. Kam, Biopolymers 20 , 231 (1981).

${ }^{66}$ T. Odijk, J. Polym. Sci. Polym. Phys. Ed. 15, 477 (1977).

${ }^{67} \mathrm{~J}$. Skolnick and M. Fixman, Macromolecules 10, 944 (1977).

${ }^{68}$ J. M. Schurr and S. A. Allison, Biopolymers 20, 251 (1981).

${ }^{69}$ T. Odijk, Biopolymers 18, 311 (1979).

${ }^{70} \mathrm{C}$. R. Cantor and P. R. Schimmel, Biophysical Chemistry (Freeman, San Francisco, 1980), p. 1155. 
${ }^{71}$ D. M. Crothers and B. H. Zimm, J. Mol. Biol. 12, 525 (1965).

${ }^{72}$ M. Becker, Ph.D. thesis, California Institute of Technology, 1981.

${ }^{73}$ D. Genest and P. Wahl, Biochim. Biophys. Acta 521, 502 (1978).
${ }^{74}$ C. Tsai, C. S. Jain, and H. M. Sobell, Proc. Natl. Acad. Sci. U.S. A. 72, 628 (1975).

${ }^{75}$ P. H. Bolton and D. R. Kearns, Nucleic Acids Res. 5, 4891 (1978).

${ }^{76}$ D. E. Graves, C. L. Watkins, and L. W. Yielding, Biochemistry 20, 1887 (1981). 